\title{
Time and Frequency Domain Identification of Seismically Isolated Structures: Advantages and Limitations
}

\author{
G. Kampas ${ }^{1 \mathrm{a}}$ and N. Makris*1b \\ ${ }^{1}$ :Division of Structures, Department of Civil Engineering, University of Patras, Greece
}

\begin{abstract}
This paper investigates the effectiveness of widely used identification methods to identify the response of seismically isolated structures supported on bearings with bilinear behavior. The paper shows that while both time domain and frequency domain methods predict with high accuracy the modal characteristics of structures isolated by linear isolation system, their performance degrades appreciably when the isolation system exhibits bilinear behavior even when its strength assumes moderate values (say $5 \%$ of the weight). The paper also shows that the natural period of isolated structure that results from bilinear isolation systems can be satisfactorily predicted with wavelet analysis.
\end{abstract}

Keywords: seismic isolation; bilinear behavior; system identification; wavelet analysis.

\section{Introduction}

In the area of civil engineering there has been an appreciable growth in infrastructure projects such as bridges, dams, pipelines, oil platforms and other industrial facilities. The vital function of these facilities in association with the need to remain operational after earthquake shaking, severe wind storms or other natural or man-induced hazards has fostered the development and implementation of structural health monitoring technologies. Such technologies have evolved appreciably both in the frequency and time domain and can identify not only the modal parameters of the system of interest (Aström and Bohlin 1965, Ewins 1984, Ljung 1987, Maia and Silva 2001, Maia 2001, Peeters and Ventura 2003, Beck and Beck 1985, Arici and Mosalam 2005, 2006, Ali and Okabayashi 2011, Shrikhande 2011), but also the location of localized damage (Kim and Melhem 2003, Wang et al. 1996, Adeli and Jiang 2006, Hearn and Testa 1991, Salawu 1997).

This paper is concerned with the effectiveness of widely used structural identification methods to identify the modal parameters from the response of seismically isolated structures such as bridges (Olmos and Roesset 2010). While, according to most design codes (AASHTO 1991, NZMWD 1983, FEMA 1997, Eurocode 2009), seismic isolation is understood as a flexible interface that merely lengthens the "vibration period" of the

a: PhD candidate, Email: gkampas@upatras.gr

b: Corresponding author. Professor, Email: nmakris@upatras.gr 
structure; most practical isolation systems comprising either with lead rubber bearings or spherical sliding bearings exhibiting bilinear behavior that may challenge the effectiveness of identification methods such as the Prediction Error Method (PEM) which is a time domain method, the Peak Picking Method (PPM) which is a frequency domain method and the wavelet transform method (WTM) which essentially concentrates on extracting the most energetic component of the response.

The paper shows that while both time domain and frequency domain methods predict with outstanding accuracy the modal characteristics of structures isolated on linear isolation systems, their performance degrades appreciably when the isolation system exhibits bilinear behavior.

\section{Problem Statement}

The PEM, PPM and the WTM are applied to extract the modal periods and damping ratios of the 2-dof structure shown in Figure 1. The attractive feature of this simple model structure is that for the case where the isolation bearings exhibit a linear viscoelastic behavior, the modal characteristics are offered by known closed form expressions (Kelly 1997). With reference to Figure 1, a typical value for the period of isolated superstructure," $T_{b}=2 \pi / \omega_{b}=2 \pi \sqrt{\left(m_{s}+m_{b}\right) / k_{b}}=2.4 s$; whereas, a typical value for the period of the fixed base superstructure is $T_{s}=2 \pi / \omega_{s}=0.4 \mathrm{~s}$. The corresponding values of damping ratios are $\xi_{b}=\frac{C_{b}}{2\left(m_{s}+m_{b}\right) \omega_{b}}=0.1$ and $\xi_{s}=\frac{C_{s}}{2\left(m_{s}+m_{b}\right) \omega_{s}}=0.03$. Furthermore, the mass ratio of the system $\gamma=\frac{m_{s}}{m_{s}+m_{b}}=0.6$. For this case where the behavior of the isolation bearings is linear viscoelastic, modal analysis gives (Kelly 1997)

$$
\begin{aligned}
& \omega_{1}^{2}=\omega_{b}^{2}\left(1-\gamma \frac{\omega_{b}^{2}}{\omega_{s}^{2}}\right) \\
& \omega_{2}=\frac{\omega_{s}^{2}}{1-\gamma}\left(1+\gamma \frac{\omega_{b}^{2}}{\omega_{s}^{2}}\right) \\
& \xi_{1}=\xi_{b}\left(1-\frac{3}{2} \gamma \frac{\omega_{b}^{2}}{\omega_{s}{ }^{2}}\right)
\end{aligned}
$$




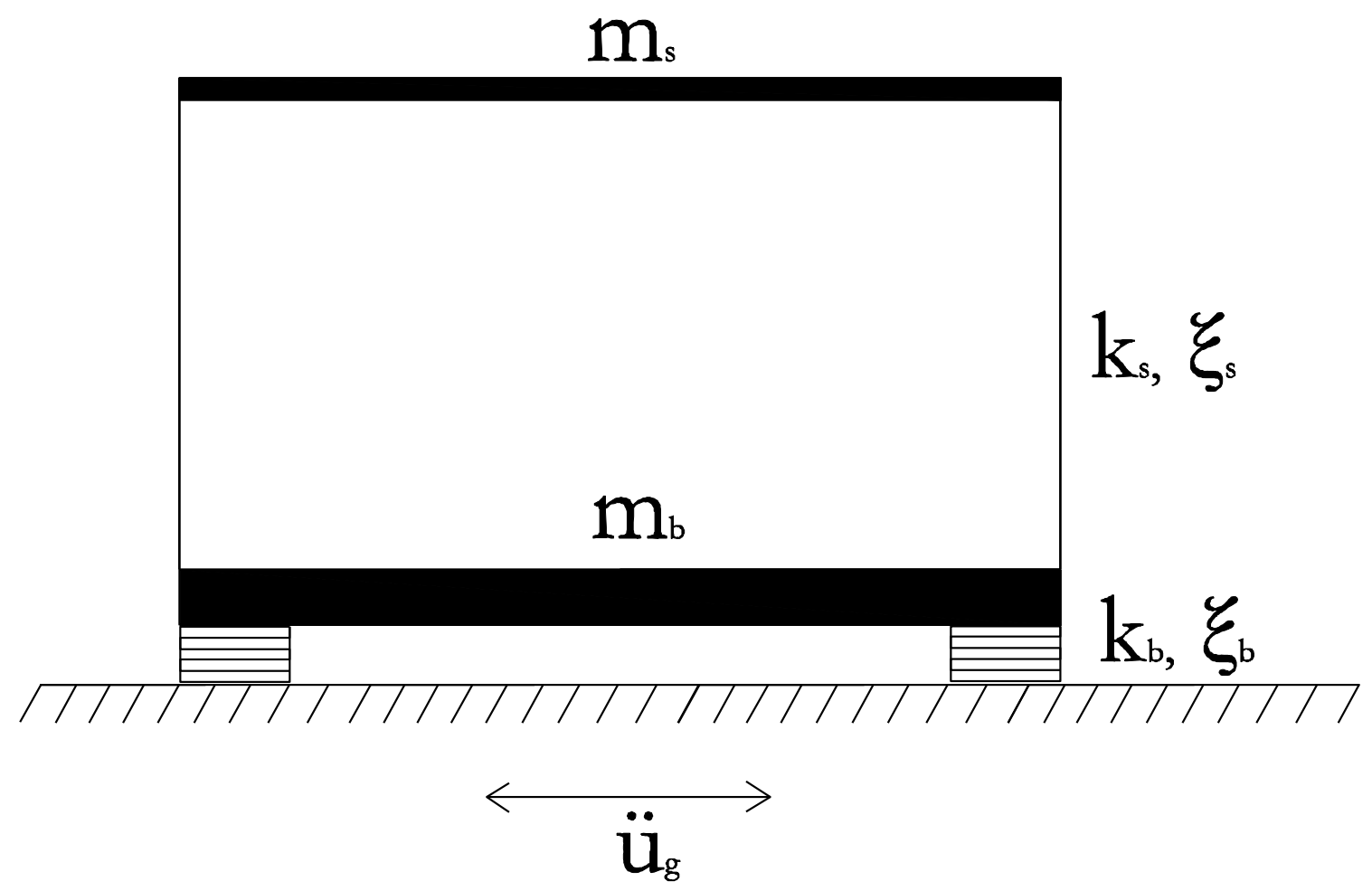

Figure 1. A one bay frame supported on a seismic isolated base.

$$
\xi_{2}=\frac{1}{\sqrt{1-\gamma}}\left(\xi_{s}+\gamma \xi_{b} \frac{\omega_{b}}{\omega_{s}}\right)
$$

From equations (1) to (4) and the aforementioned values of $\omega_{b}=2 \pi / T_{b}=2.618 \mathrm{rad} / \mathrm{s}$, $\omega_{s}=2 \pi / T_{s}=15.708 \mathrm{rad} / \mathrm{s}, \quad \xi_{b}=0.1$ and $\xi_{s}=0.03$, the theoretical modal periods and damping ratios are $T_{1}=2.42 s, T_{2}=0.25 s, \xi_{1}=0.1$ and $\xi_{2}=0.032$. These are the target values expected to be identified by the three identification methods examined in this paper.

When the behavior of the isolation system is bilinear, $k_{b}$ is now the second slope of the bilinear system; whereas $Q$ is the strength of the system -that is the force that corresponds to zero deformation in the hysteresis loop, while $u_{y}$ is the yield displacement as shown in Figure 2. With reference to Figure 2, the first slope, $k_{0}$ of the bilinear system is given by

$$
k_{0}=\frac{Q}{u_{y}}+k_{b}
$$

and the pre-yielding period of the bilinear system is 


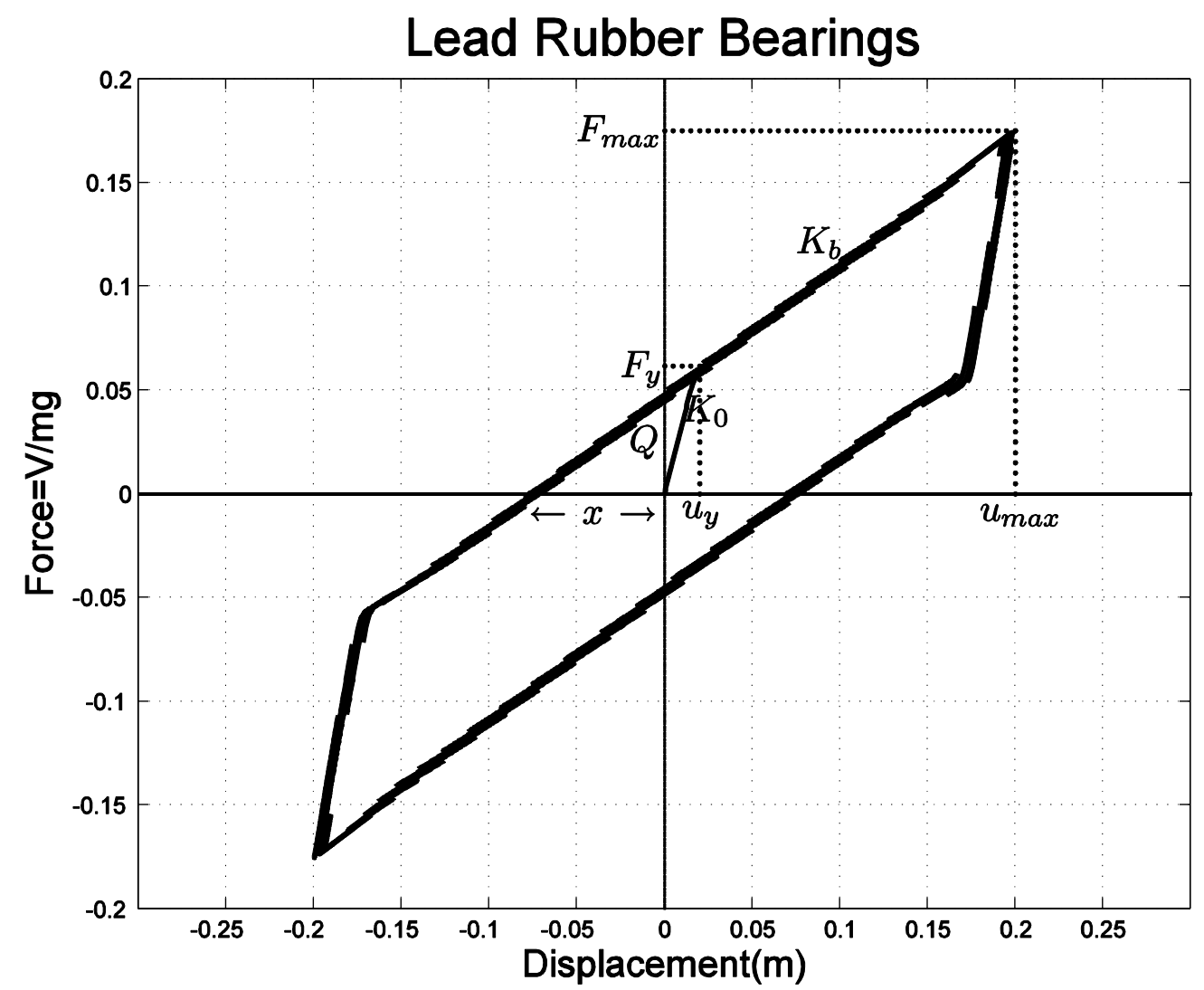

Figure 2. Schematic of a force-displacement loop with bilinear behavior.

$$
T_{0}=\sqrt{\frac{1}{\frac{1}{T_{b}^{2}}+\frac{Q}{4 \pi^{2}\left(m_{s}+m_{b}\right) u_{y}}}}
$$

Typical values of the yield displacement $u_{y}$ for lead rubber bearings (LRB) is $u_{y}=2 \mathrm{~cm}=0.02 \mathrm{~m}$; whereas, for spherical sliding bearings $u_{y}=0.025 \mathrm{~cm}=0.00025 \mathrm{~m}$ (Constantinou et al. 1990, Mokha et al. 1990). In this study we assume a typical value of the normalized strength of the bearings $\frac{Q}{m_{s}+m_{b}}=0.05 \mathrm{~g}$. The system parameters given above together with the pre-yielding period as offered by equation (6) are summarized in Table 1.

Clearly, when the behavior of the isolation system is bilinear, the response of the isolated structure departs from the linear idealization. Nevertheless, when the excitation is strong enough so that the behavior of the bearing enters appreciably the second slope there is evidence from dimensional analysis studies, that it is the second slope, $k_{b}$, that governs the 
Table 1. Parameters of the bilinear model associated with the LRB and SSB assumed in this study.

$$
T_{b}(\mathrm{sec}) \quad \frac{Q}{m_{s}+m_{b}}(g) \quad u_{y}(m) \quad T_{0}(\mathrm{sec})
$$
LRB
0.05
0.025
1.1212
SSB
2.4
0.05
0.00025
0.1266

response (Makris and Black 2004, Makris and Vassiliou 2011).Part of the scope of this study is to identify via system identification techniques the dominant periods and associated "equivalent linear" damping ratios that emerge from the time history responses of the simple structural model shown in Figure 1 when supported on an isolation system with bilinear behavior.

\section{Response Histories}

The response histories of the $2 \mathrm{DOF}$ system shown in Figure $1\left(u_{b}, \dot{u}_{b}, u_{s}, \dot{u}_{s}\right)$ when isolated by linear viscoelastic bearings or on bilinear bearings are computed via direct integration of the equations of motion (Kelly 1997, Makris and Chang 2000, Makris and Black 2004). The 2DOF system structure is subjected to four well known strong historic records listed in Table 2.

Table 2. Information pertinent to the strong records selected for this study.

$\begin{array}{ccccc}\text { No } & \text { Earthquake } & \text { Record Station } & \text { Mw } & \text { PGA (g) } \\ 1 & \text { 1971 San } & \text { Pacoima Dam } & 6.6 & 1.226 \\ \text { Fernando } & \text { (St. 164) } & & \\ 2 & \text { 95 Erzincan } & 6.9 & 0.515 \\ 3 & \text { (N-S comp) } & & 0.571 \\ & & \text { Jensen Filter } & 6.7 & \\ 4 & \text { Station (St. 022) } & & 0.476\end{array}$


Figures 3 and 4 plot the acceleration time histories of the four earthquakes listed in Table 2 together with the acceleration responses of the base above isolators and the superstructure when isolated with lead rubber bearings. On the right of Figure 3 and 4 the forcedisplacement loops of the isolators are shown. The computed acceleration histories $\ddot{u}_{b}$ and $\ddot{u}_{s}$ are used in the system identification study reported herein to extract the modal periods and damping ratios of the isolated structures and the identified values are compared with the theoretical values. The corresponding response histories when the structure is isolated by spherical sliding bearings with the same strength $Q /\left(m_{s}+m_{b}\right)=\mu=5 \%$ and the same second slope, $k_{b}$ are shown in Figures 5 and 6.

\section{Time Domain Identification Methods}

In the last decades several time domain techniques were advanced for system identification purposes. Some of them are the Prediction Error Method, the Autoregressive Moving Average methods (ARMA, ARMAX, ARMAV), the Ibrahim Time Domain Method, the Eigensystem Realization Algorithm, the IRF Driven/Covariance-Driven Subspace Identification Method, Extended Kalman Filter Algorithm and others (Peeters and Ventura 2003, Allamang and Brown 1998, Maia and Silva 2001, Arici and Mosalam 2005, among others). One of the most efficient, powerful and accurate method that can be applied for the identification of modal parameters is the Prediction Error Method (PEM). It initially emerged from the maximum likelihood framework of Aström and Bohlin (1965), and was advanced to become popular to system identification engineers as a MATLAB (2002) identification toolbox was developed following the theory by Ljung $(1987,1994,2002)$.

\subsection{THE PREDICTION ERROR METHOD (PEM)}

Prediction error methods belong to a broad family of parameter estimation methods that can be applied to arbitrary model parameterizations (Ljung 2002). Thus, given an output $y(t)$ due to an input $u(t)$ at time $t$, the target is to identify the parameters of the selected model. The recordings are discrete in time and let $Z^{N}=\{u(1), y(1), u(2), y(2), \ldots u(N), y(N)\}$ be all the past data recorded up to time $t=N$. However, the methods can also deal with continuous-time models. The basic idea that lies behind these methods is that the model can be described as a predictor of the next output point as a function of the past history, 


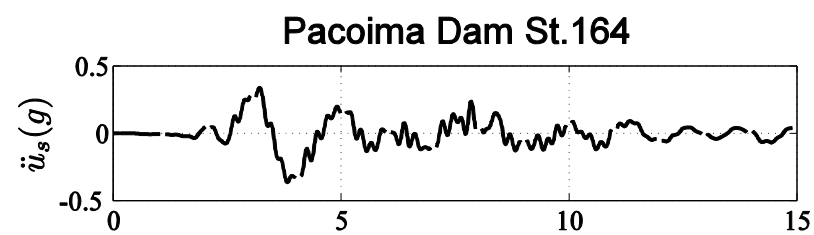

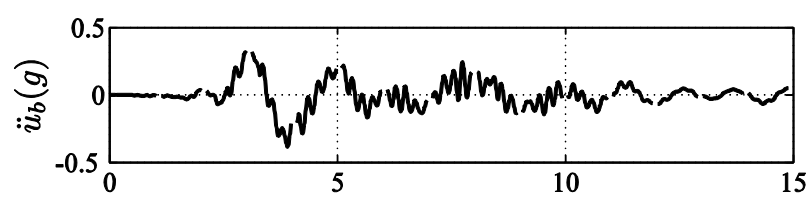

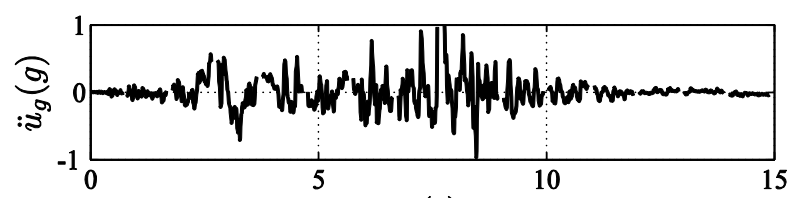

$\mathrm{t}(\mathrm{s})$
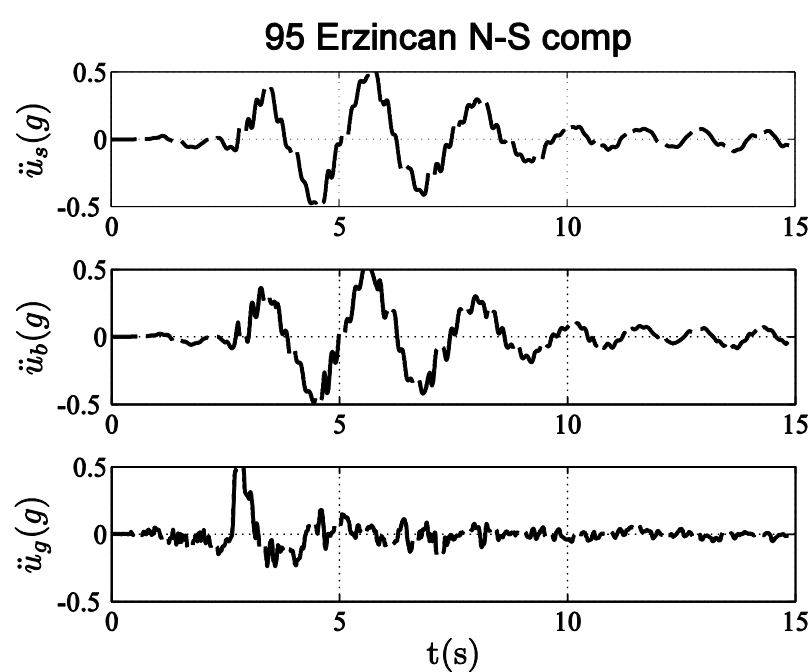
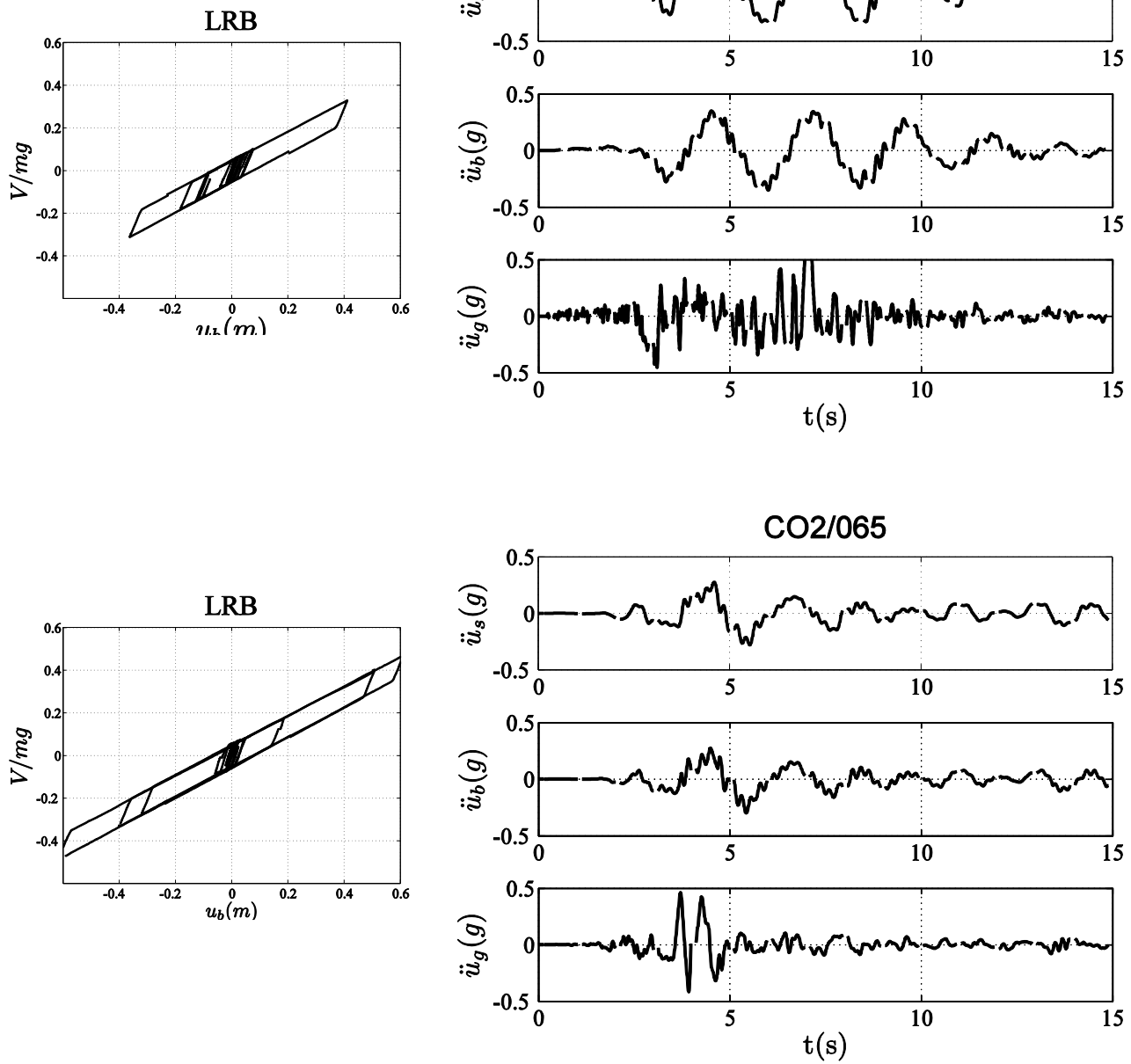
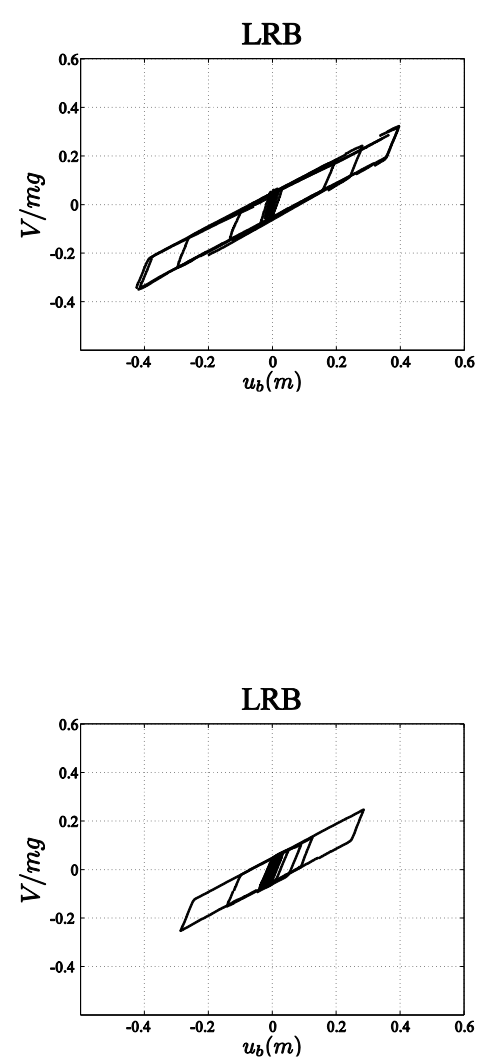

Figure 3. Ground, above isolators and superstructure acceleration histories together with the corresponding force-displacement loops of the 2DOF system shown in Figure 1 supported on lead rubber bearings when subjected to the four recorded motions listed in Table 2. 

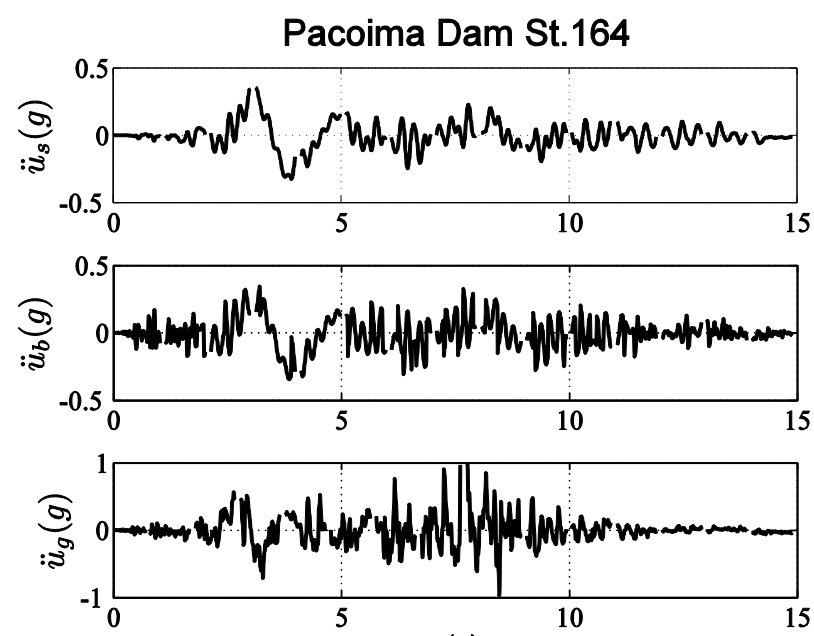

$\mathrm{t}(\mathrm{s})$
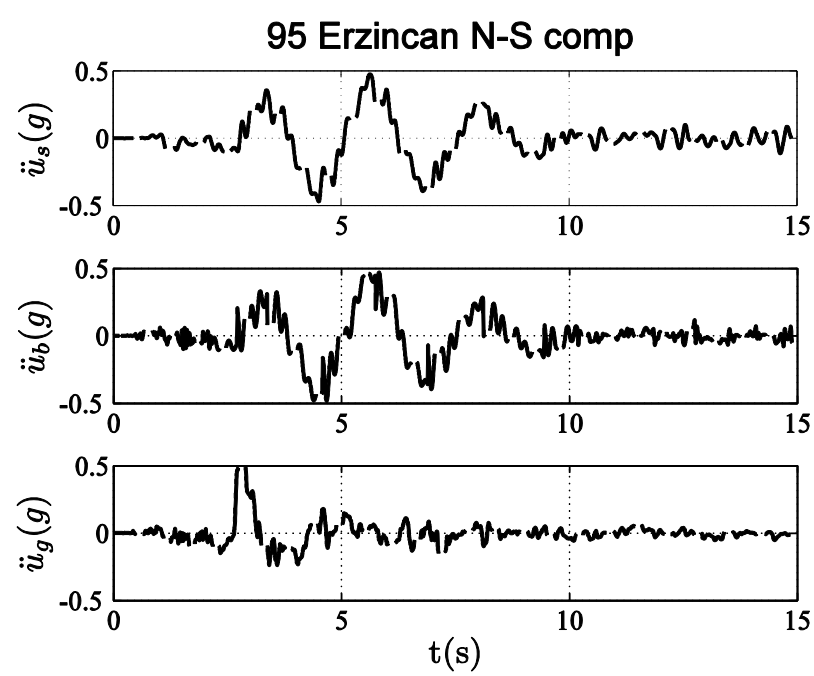
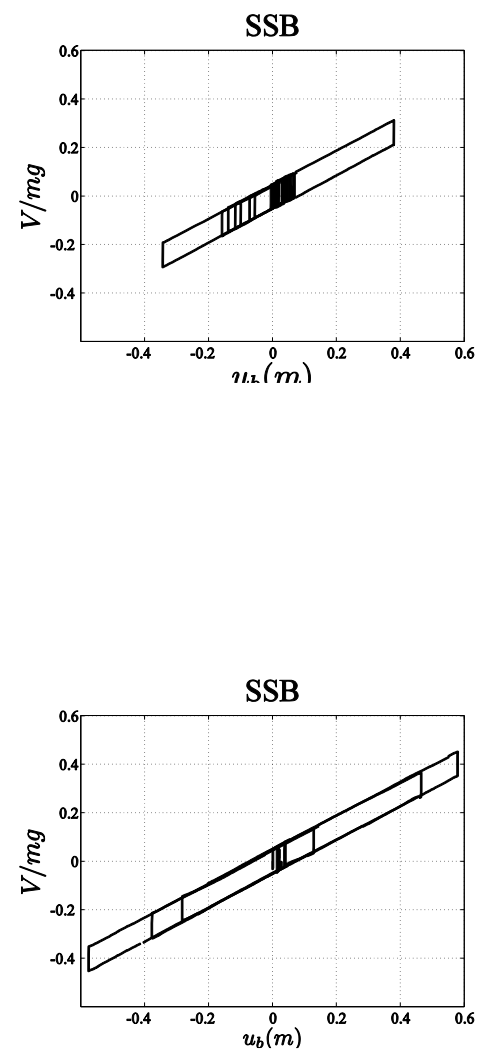
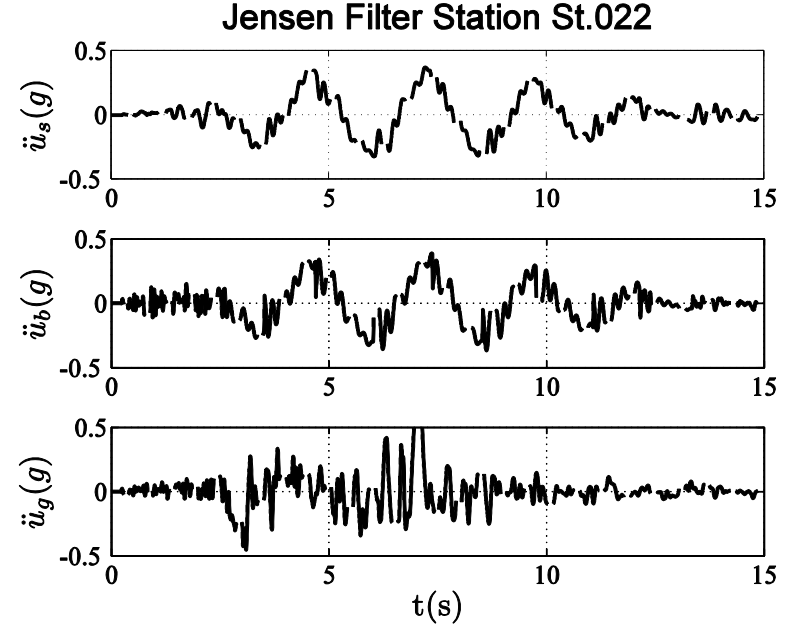

$\mathrm{t}(\mathrm{s})$
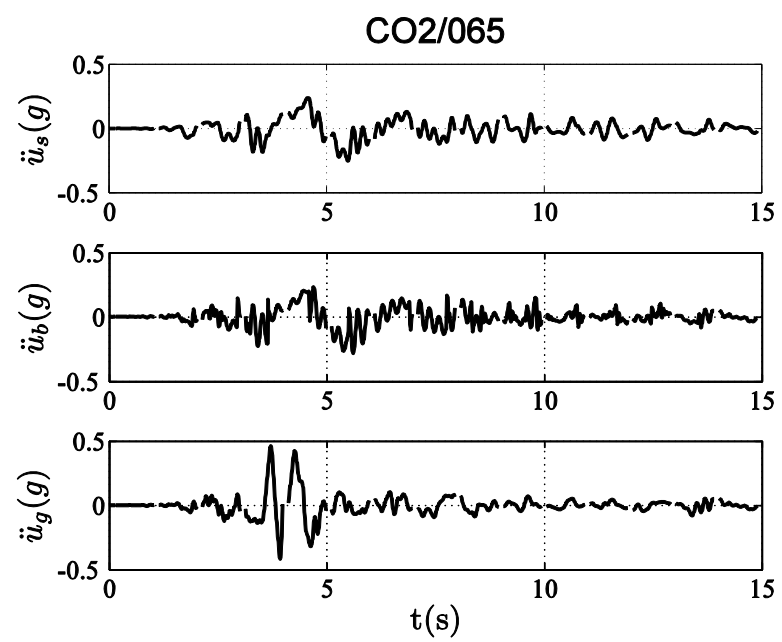

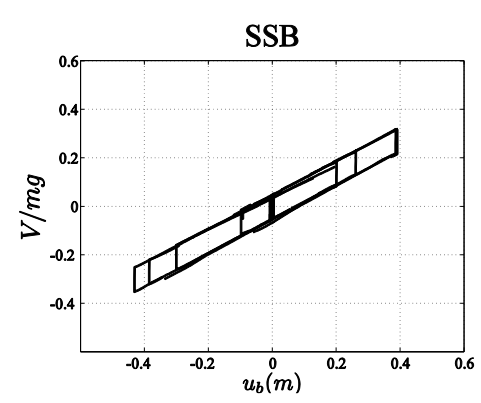

Figure 4. Ground, above isolators and superstructure acceleration histories together with the corresponding force-displacement loops of the 2DOF system shown in Figure 1 supported on spherical sliding bearings when subjected to the four recorded motions listed in Table 2. 


$$
\hat{y}_{m}(t \mid t-1)=f\left(Z^{t-1}\right)
$$

where $\hat{y}_{m}(t \mid t-1)$ accounts for the predictor, and $f\left(Z^{t-1}\right)$ for the chosen, arbitrary function of past data. The next conceptual step is to parameterize the predictor using a parameter vector, $\bar{\theta}$,

$$
\hat{y}(t \mid \boldsymbol{\theta})=f\left(Z^{t-1}, \boldsymbol{\theta}\right)
$$

The method's final outcome is an estimate of the parameter vector $\boldsymbol{\theta}, \theta_{N}$, according to the minimization of an appropriate norm which represents the distance, $V_{N}(\boldsymbol{\theta})$, between the predicted output $\{y(1 \mid \boldsymbol{\theta}), \ldots, y(N \mid \boldsymbol{\theta})\}$ and the recorded output $\{y(1), \ldots, y(N)\}$ :

$$
V_{N}(\boldsymbol{\theta})=\sum_{t=1}^{N} l(y(t)-\hat{y}(t \mid \boldsymbol{\theta}))=\sum_{t=1}^{N} l\left(y(t)-f\left(Z^{t-1}, \boldsymbol{\theta}\right)\right)
$$

where $l=\|y(t)-\hat{y}(t \mid \boldsymbol{\theta})\|^{2}$ is the suitable distance measure. The parameter vector $\hat{\theta}_{N}$ is calculated by minimizing the above norm,

$$
\hat{\theta}_{N}=\arg \min _{\theta} V_{N}(\boldsymbol{\theta})
$$

\subsubsection{State-Space Representation of the linear model}

The mathematical model of choice to represent dynamic systems when applying PEM is the state-space model. State-space modeling is most common in structural dynamics, as it reduces the second order differential equation of motion to a system of first order differential equations (Akira 2003, Lembregts et al. 1990).

Thus, the relationship between the input and output signals is written as a system of first order differential equations using a state vector $\bar{x}(t)$ :

$$
\begin{aligned}
\dot{\boldsymbol{x}}(t) & =[F(\boldsymbol{\theta})] \boldsymbol{x}(t)+[G(\boldsymbol{\theta})] \boldsymbol{u}(t) \\
\boldsymbol{y}(t) & =[H(\boldsymbol{\theta})] \boldsymbol{x}(t)+[D(\boldsymbol{\theta})] \boldsymbol{u}(t)
\end{aligned}
$$

where $\boldsymbol{x}(t)$ is the state vector, $\boldsymbol{y}(t)$ is the output vector and $\boldsymbol{u}(t)$ is the input vector. $[F(\boldsymbol{\theta})],[G(\boldsymbol{\theta})]$ are matrices of appropriate dimensions $(n \times n$ and $n \times m$ respectively for an $n$-dimensional state and $m$ dimensional input). The same is true for $[H(\boldsymbol{\theta})],[D(\boldsymbol{\theta})]$ respectively. $\boldsymbol{\theta}$ represents the unknown parameter vector and the overdot denotes differentiation with respect to time (Ljung 1987, 2002). 
In our case, the state vector's components are the displacement and velocity vectors, $\boldsymbol{x}(t)=\left[\begin{array}{ll}\boldsymbol{u}(t)^{T} & \dot{\boldsymbol{u}}(t)^{T}\end{array}\right]^{T}:$

$$
\begin{aligned}
& \dot{\boldsymbol{x}}(t)=\left[A_{C}\right] \boldsymbol{x}(t)+\left[B_{C}\right] \ddot{u}_{g}(t) \\
& \boldsymbol{y}(t)=\left[C_{C}\right] \boldsymbol{x}(t)+\left[D_{C}\right] \ddot{u}_{g}(t)
\end{aligned}
$$

where,

$$
\begin{aligned}
& {\left[A_{C}\right]=\left[\begin{array}{cc}
0 & I \\
-M^{-1} K & -M^{-1} C_{D}
\end{array}\right], \quad\left[B_{C}\right]=\left[\begin{array}{c}
0 \\
-M^{-1} B_{f}
\end{array}\right]} \\
& {\left[C_{C}\right]=\left[\begin{array}{ll}
-M^{-1} K & -M^{-1} C_{D}
\end{array}\right], \quad\left[D_{C}\right]=\left[-M^{-1} B_{f}\right]}
\end{aligned}
$$

while $\left[A_{C}\right]$ is the state transition matrix, $\left[B_{C}\right]$ is the input influence matrix, $\left[C_{C}\right]$ is the output influence matrix, $\left[D_{C}\right]$ is the direct transmission term, and are composed of mass matrix $M$, stiffness matrix $K$, damping matrix $C_{D}$ and influence matrix $B_{f}$. $\ddot{u}_{g}(t)$ signifies the strong ground motion input excitation (Arici and Mosalam 2005, 2006). In this study the recordings are accelerations, as the instruments across the bridge are accelerometers, thus the output $\boldsymbol{y}(t)$ is the acceleration, $\ddot{u}(t)$.

The eigenvalues of the system can be deduced from the eigenvalue problem:

$$
\left[A_{C}\right] \boldsymbol{p}=\gamma_{i} \boldsymbol{p}
$$

where $\bar{p}$ is the mode vector and $\gamma_{i}$ are the complex eigenvalues of the system,

$$
\gamma_{i}=\sigma_{i} \pm i \omega_{i}=-\xi_{i} \omega_{i} \pm i \omega_{i} \sqrt{1-\xi_{i}^{2}}
$$

where $\omega_{i}$ is the undamped natural frequency and $\xi_{i}$ is the damping ratio of the $i^{\text {th }}$ mode. The complex eigevalues have the above form assuming that damping has a proportional viscous form.

\subsubsection{Continuous to discrete time model}

The above formulation refers to continuous time models. For discrete time models and by using zero order hold method (Juang 1994), equation (12) becomes 


$$
\begin{aligned}
& \boldsymbol{x}(t+1)=[A] \boldsymbol{x}(t)+[B] \ddot{u}_{g}(t)+w(t) \\
& \boldsymbol{y}(t)=\left[C_{C}\right] \boldsymbol{x}(t)+\left[D_{C}\right] \ddot{u}_{g}(t)+e(t)
\end{aligned}
$$

where $[A]=e^{\left[A_{C}\right] \Delta t}$ and $[B]=\int_{0}^{\Delta t} e^{\left[A_{C}\right] \tau} d \tau\left[B_{C}\right]$ with $\Delta t$ representing the sample time interval. The $\left[C_{C}\right],\left[D_{C}\right]$ matrices remain the same as in the continuous model, while $w(t)$ accounts for the process noise and $e(t)$ for the measurement noise. The eigenvalue problem (14)becomes

$$
[A] \boldsymbol{p}=\lambda_{i} \boldsymbol{p} \Rightarrow e^{\left[A_{C}\right] \Delta t} \boldsymbol{p}=\lambda_{i} \boldsymbol{p}
$$

from which,

$$
\lambda_{i}=e^{\gamma_{i} \Delta t} \text { or } \gamma_{i}=\frac{\ln \left[\lambda_{i}\right]}{\Delta t}
$$

Thus, by adopting the discrete eigenvalues $\lambda_{i}$, and by using the transformation given by equation (18) one obtains the continuous complex eigenvalues. After obtaining the complex eigenvalues, the next step is to determine the natural undamped frequency and damping ratio for the $i^{\text {th }}$ mode (Arici and Mosalam 2005):

$$
\omega_{i}=\sqrt{\gamma_{i} \times \gamma_{i}^{*}}, \quad \xi_{i}=-\frac{\operatorname{Re}\left(\gamma_{i}\right)}{\omega_{i}}
$$

The abovementioned procedure is implemented via four different approaches; SISO approach (single input/single output) assuming that the system has one single input and one single output, SIMO approach (single input/multi output) assuming that the system has a single input and multi outputs, MISO approach (multi input/single output) assuming that the system has multi inputs and a single output and MIMO (multi input/multi output) assuming that the system has multi inputs and multi outputs.

Furthermore, SISO approach can be applied in complex dynamic systems for retrieving local dynamic properties of sub-elements of the total structure, MISO is an extended SISO accounting also for different input motions, SIMO is applied for deducing more general dynamic properties of the total structure and MIMO is the most essential approach for deducing the global characteristics of the structure. In this paper, SISO is the most appropriate approach since it is important to retrieve information from both degrees of freedom separately. 


\subsection{IDENTIFICATION OF MODAL PROPERTIES OF THE 2DOF SYSTEM WITH PEM}

The effectiveness of the Prediction Error Method (PEM) in identifying the modal characteristics of the 2DOF system shown in Figure 1 isolated using linear viscoelastic bearings is presented in Figure 5. Both modal periods $T_{1}$ and $T_{2}$ are predicted with remarkable accuracy for all four earthquake excitations by processing the acceleration responses either of the isolated base, $\ddot{u}_{b}(t)$, or of the superstructure $\ddot{u}_{s}(t)$. The same is true for the predicted damping ratios which are very close to the theoretical values.

The effectiveness of PEM in identifying the modal parameters of the 2DOF system shown in Figure 1 isolated using lead rubber bearings is presented in Figure 6.

In the case when the bearing exhibits mild nonlinear behavior $\left(\frac{Q}{m g}=5 \%\right)$ PEM identifies satisfactorily the second modal period; yet its identification for the first modal period -that is the period of isolated structure, is poor. The same poor performance of PEM is observed in Figure 6 where PEM fails to identify the modal damping ratio values.

In the case where the 2DOF system is isolated by spherical sliding bearings, PEM is inappropriate for identifying the period of isolated structure from both recordings though the results for the second modal period are satisfying. The same stands for the modal damping ratios as shown in Figure 7.

\section{Frequency Domain Methods}

The large number of experimental programs associated with the "resonance testing" in conjunction with the availability of fast Fourier transform algorithms advanced frequency domain methods, such as the Peak-Picking Method (PPM), the Circle-Fitting Method, the Rational Fraction Polynomial Method, etc (Maia and Silva 2001, Maia 2001, Ewins 1984, among others). The most widely used frequency domain method is the Peak-Picking Method (PPM) given its directness and its flexibility to accommodate the user's intuition. However, the need for advancing other more sophisticated frequency domain methods has emerged from the need to overcome some of the limitations of the PPM (Ewins 1984, Peeters and Ventura 2003).

\subsection{THE PEAK-PICKING METHOD}

Peak-Picking Method (PPM) or Peak-Amplitude Method is one of the first methods advanced 


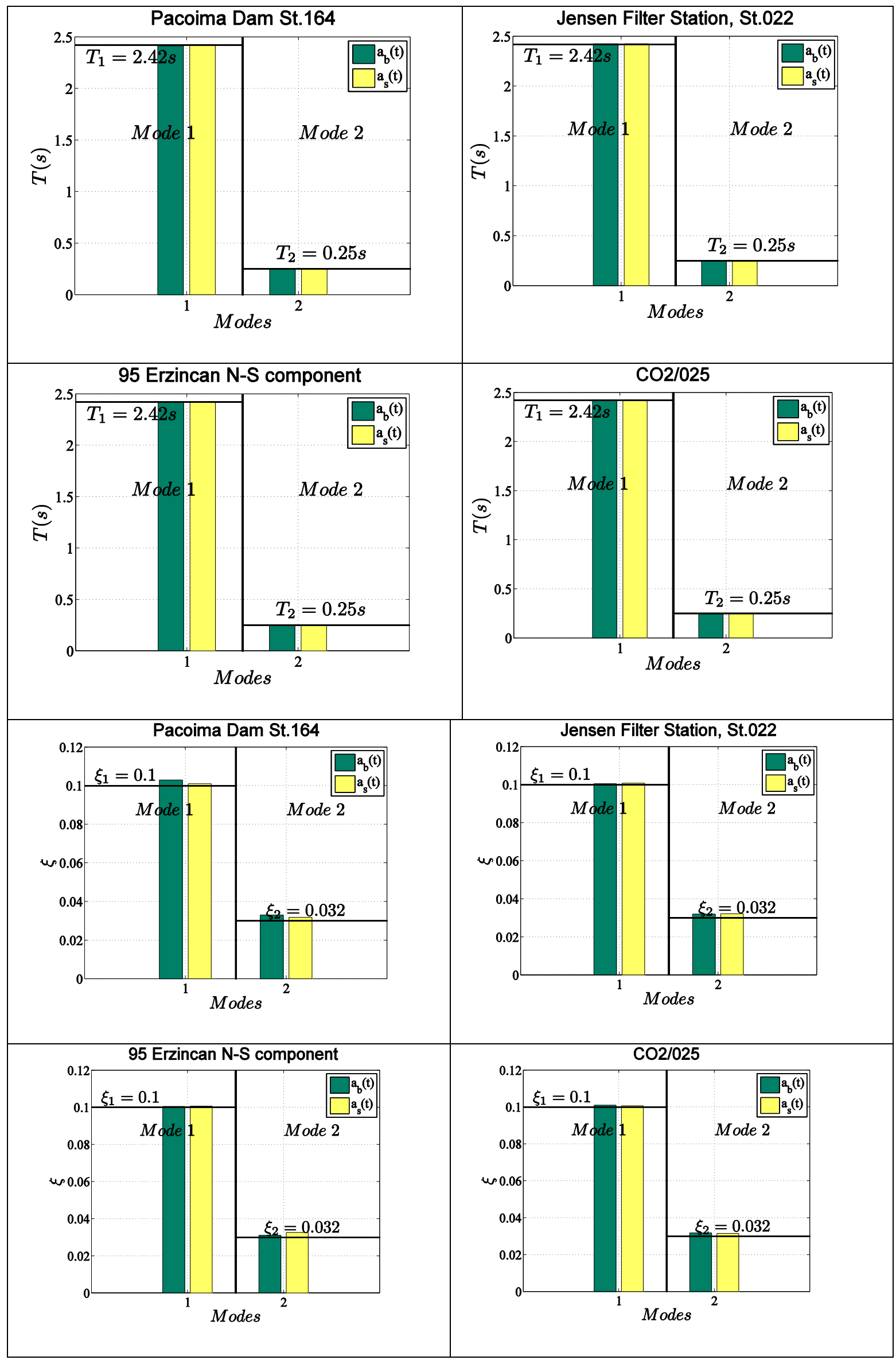

Figure 5. Eigenperiods and damping ratios of the 2DOF system shown in Figure 1 when isolated by linear viscoelastic bearings identified with the Prediction Error Method (PEM). 


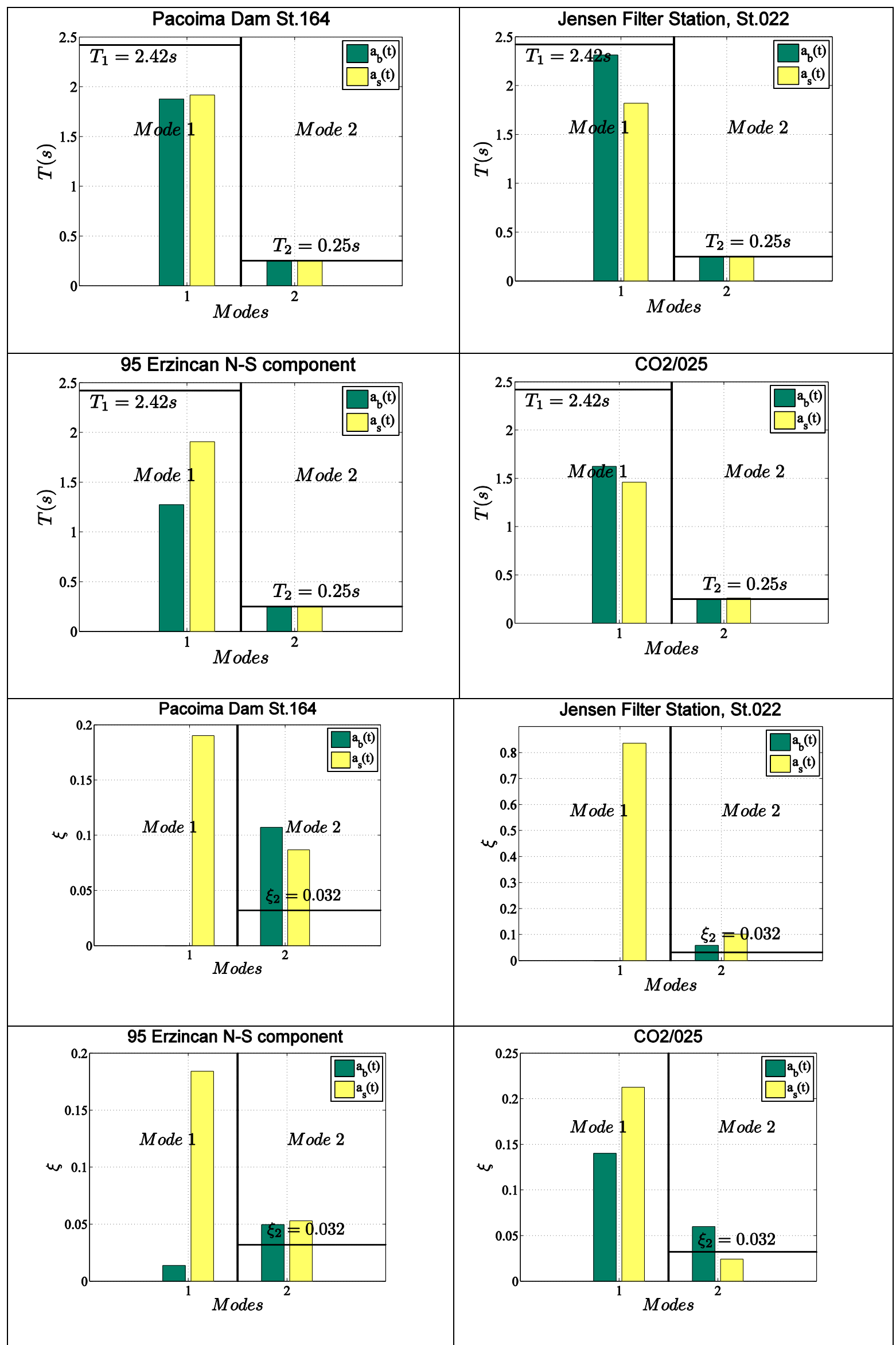

Figure 6. Eigenperiods and damping ratios of the 2DOF system shown in Figure 1 when isolated by lead rubber bearings identified with the Prediction Error Method (PEM). 


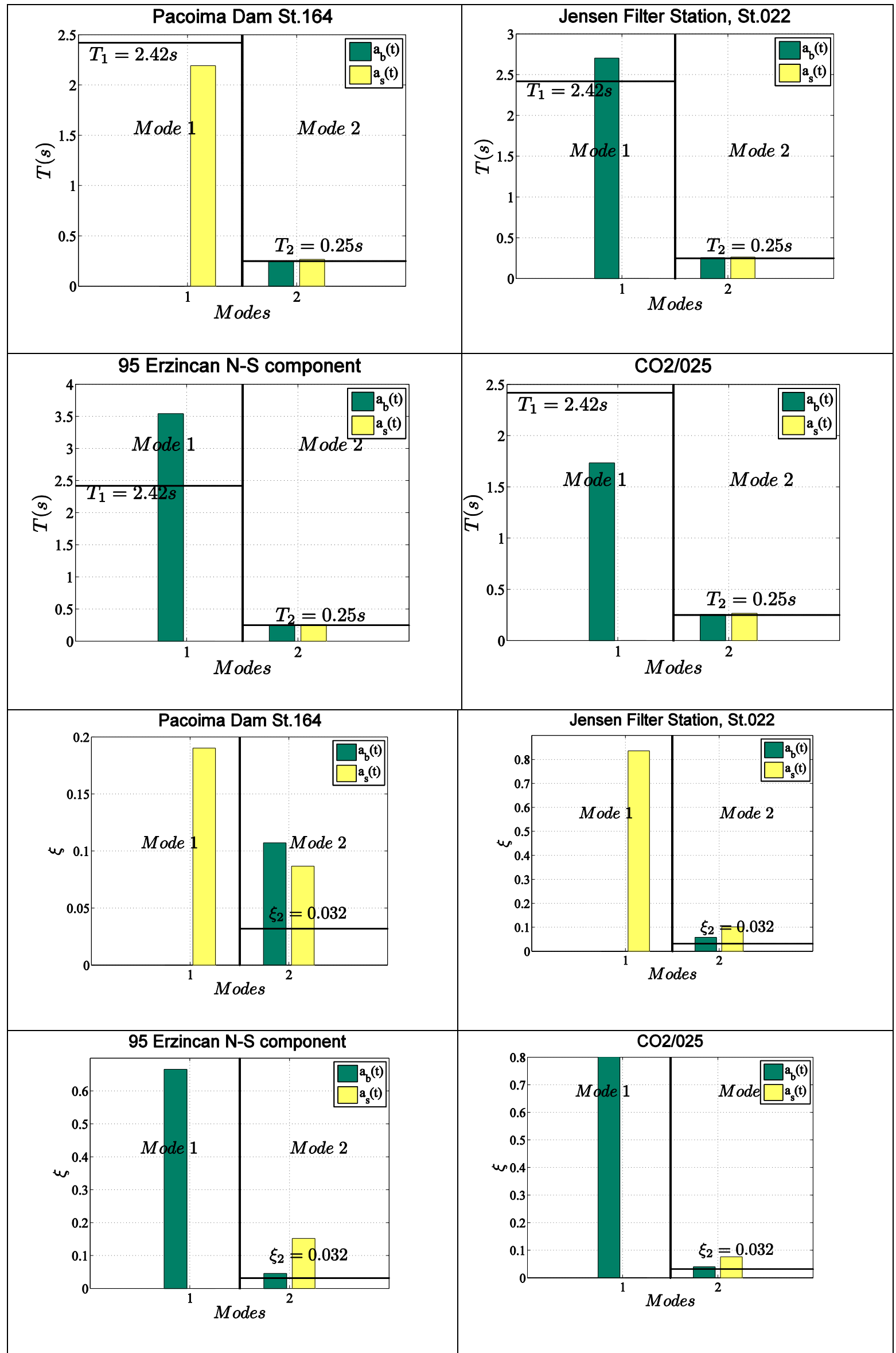

Figure 7. Eigenperiods and damping ratios of the 2DOF system shown in Figure 1 when isolated by spherical sliding bearings identified with the Prediction Error Method (PEM). 
in the frequency domain (Maia and Silva 2001, Maia 2001, Ewins 1984). Theoretically, it is probably the simplest and the most intuitive method. It is based on the assumption that in the vicinity of the resonance the total response is dominated by the contribution of the mode whose natural frequency is the closest. The method uses the frequency response function (FRF) of the structure and works adequately only when the FRF exhibits well separated modes.

The frequency that corresponds to the individual response peaks that are detected from the plot of the absolute value of the FRF signifies the natural frequency of the system,

$$
\omega_{n d}=\omega_{n} \sqrt{1-\xi_{n}^{2}}
$$

where $\omega_{n}$ is the undamped natural frequency and $\xi_{n}$ is the damping ratio. The second step is the calculation of the damping ratio using the same plot; let the maximum value of the absolute value of the FRF near resonance be noted as $|A|$, and the frequencies that correspond to $|A| / \sqrt{2}, \omega_{a}$ and $\omega_{b}$ (half power points). The damping ratio can be calculated from

$$
\xi_{n}=\frac{\omega_{a}-\omega_{b}}{2 \omega_{n}}
$$

Thus, the natural frequency and the damping ratio can be deduced from equations(20), (21) (Ewins 1984).

As aforementioned, PPM is, from a theoretical point of view, a very simple method; however in its practical application it has serious limitations. It should be applied to systems that have wellseparated modes, that are not lightly damped so that the area near resonance has the appropriate accuracy, but on the other hand, not heavily damped so that the area near resonance is not strongly influenced by other modes. Another drawback of the method is that the results are strongly affected from the frequency domain resolution -which depends on the instrument type. The above limitations are the main reasons for not depending on the results, but considering them only as initial estimates of the modal parameters (Ewins 1984).Yet, a challenging task in the application of the method is the fact that the user-engineer has to distinguish -based on his experience- the real modes from "spurious" numerical and measurement-noise peaks. 


\subsection{IDENTIFICATION OF MODAL PROPERTIES OF THE 2DOF SYSTEM WITH PPM}

Figures 8 and 9 present the transfer function of the 2 dof isolated structure for the three different isolation cases (linear viscoelastic, lead rubber, spherical sliding bearings) for the four ground motions presented in Table 2.

It is clear that in the case where the $2 \mathrm{DOF}$ system is isolated by linear viscoelastic bearings the modes are well separated and the user-engineer should have no problem in identifying the eigenperiods of the oscillator for all four ground motions, thus PPM concludes to satisfying results.

In the cases where the $2 \mathrm{DOF}$ system is isolated by lead rubber or spherical sliding bearings the mild nonlinearity that the systems exhibit in time domain is reflected as spurious spikes/noise in the frequency domain. Thus, although the modes are well separated and the results are numerical (without the corresponding noise from real measurements) and correspond to a simple 2DOF system isolated oscillator (not a complex structure), the user-engineer should not be able to distinguish clearly the eigenperiods in most cases. In such systems, PPM is inappropriate to identify the modal periods. The abovementioned observations when using PEM (time domain) and PPM (frequency domain) as modal identification methods result that the major challenge is the identification of the period of isolated structure of the system. In an effort to overcome this challenge we proceed with a time-frequency analysis to identify the period of isolated structure of the structure when supported on bearings with bilinear behavior.

\section{Time-Frequency Domain Methods}

\subsection{THE WAVELET TRANSFORM METHOD}

Over the last two decades, wavelet transform analysis has emerged as a unique new timefrequency decomposition tool for signal processing and data analysis. There is wide literature available regarding its mathematical foundation and its applications (Mallat 1999, Addison 2002 and references reported therein). Wavelets are simple wavelike functions localized in time. For

instance, the second derivative of the Gaussian distribution, $e^{-t^{2} / 2}$, known in the seismology literature as the symmetric Ricker Wavelet (Ricker 1943, 1944; and widely referred as the "Mexican Hat" wavelet, Addison 2002),

$$
\psi(t)=\left(1-t^{2}\right) e^{-t^{2} / 2}
$$


Pacoima Dam, St.164-LB
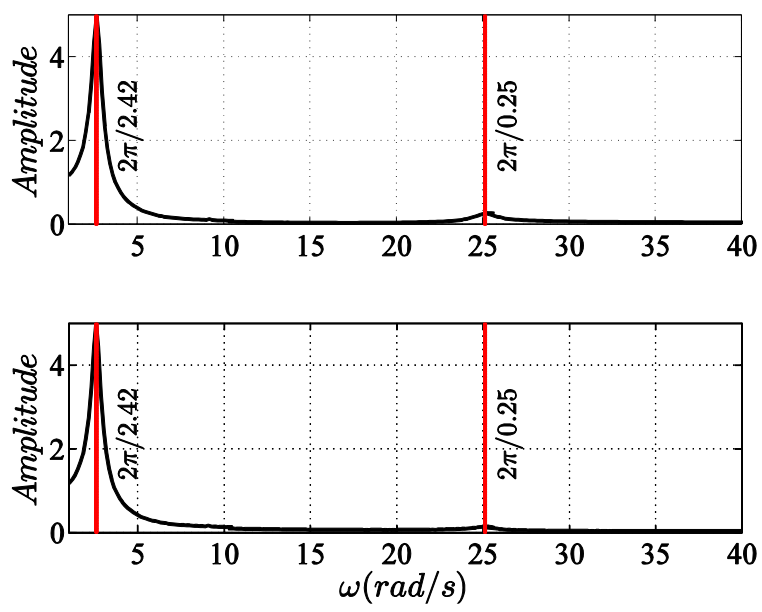

Pacoima Dam, St.164-LRB
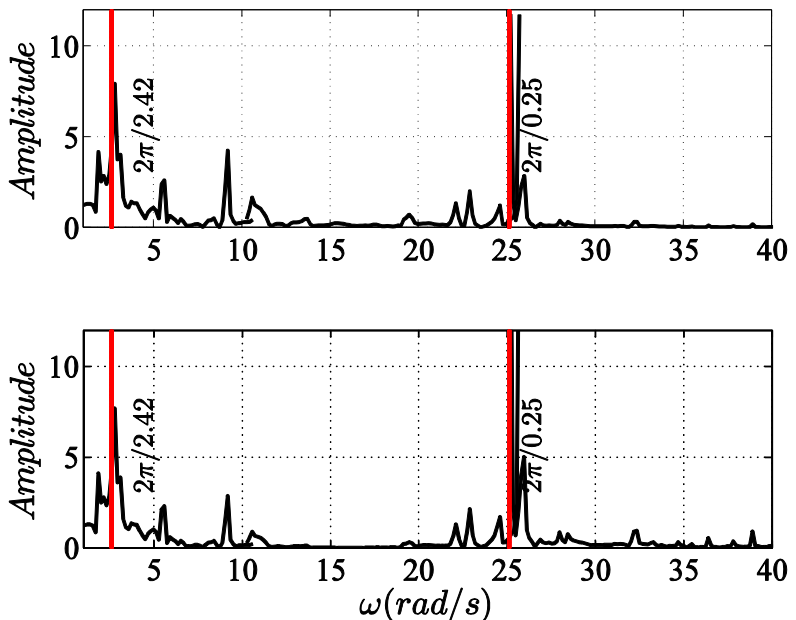

Pacoima Dam, St.164-SSB
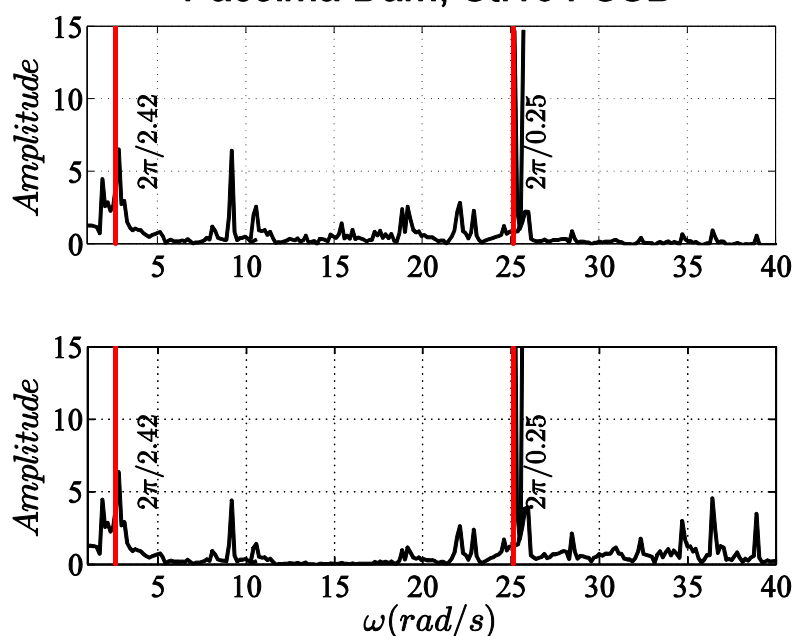

95 Erzincan, N-S comp-LB
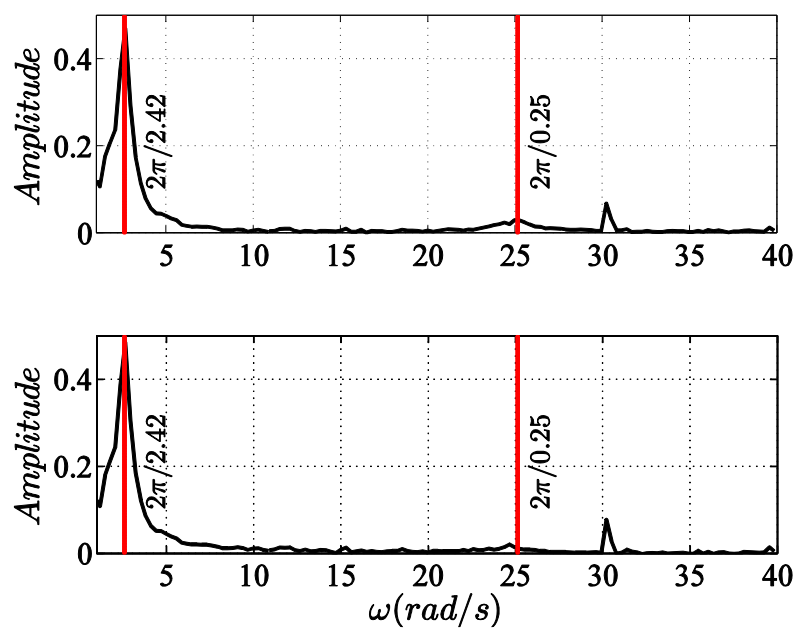

95 Erzincan, N-S comp-LRB
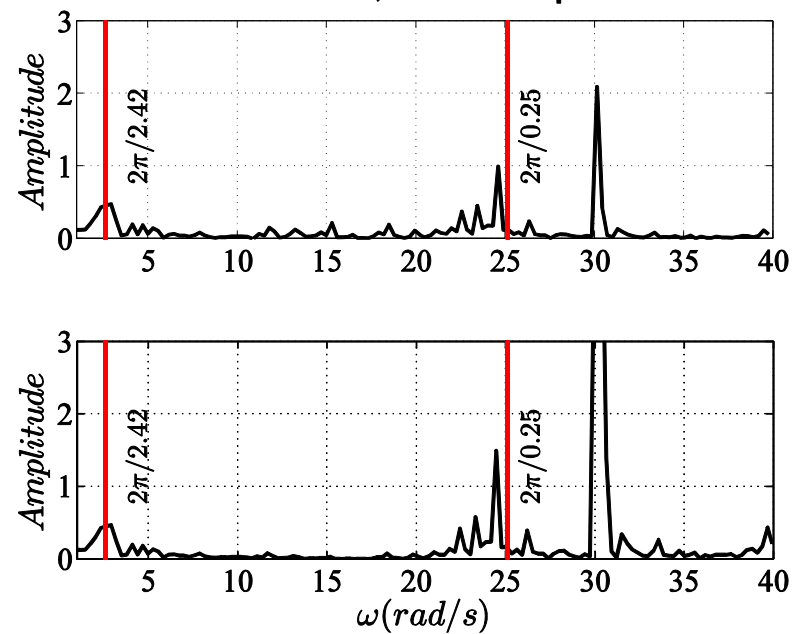

95 Erzincan, N-S comp-SSB
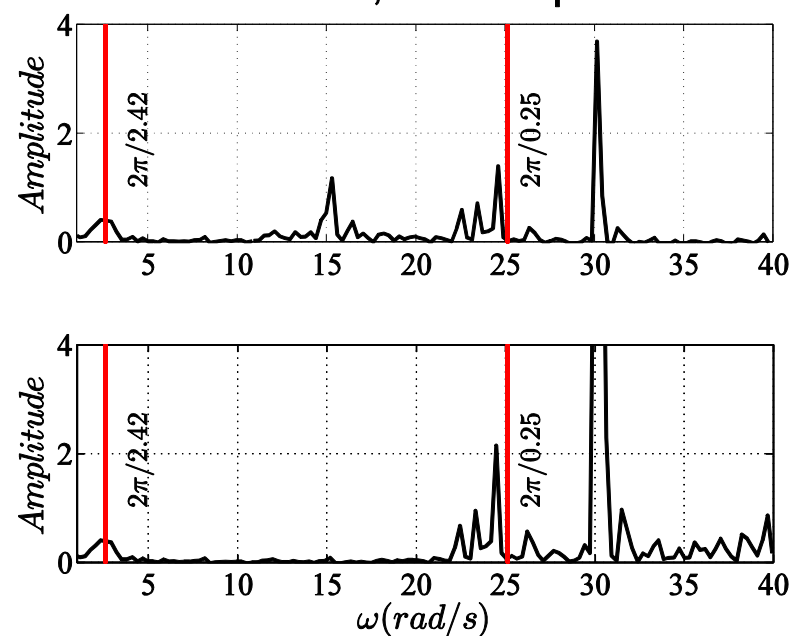

Figure 8. Transfer functions of the 2DOF system isolated by linear (top), lead rubber (middle) and spherical sliding bearings (bottom) when subjected to the San Fernando (left) and Erzincan (right) earthquake. 

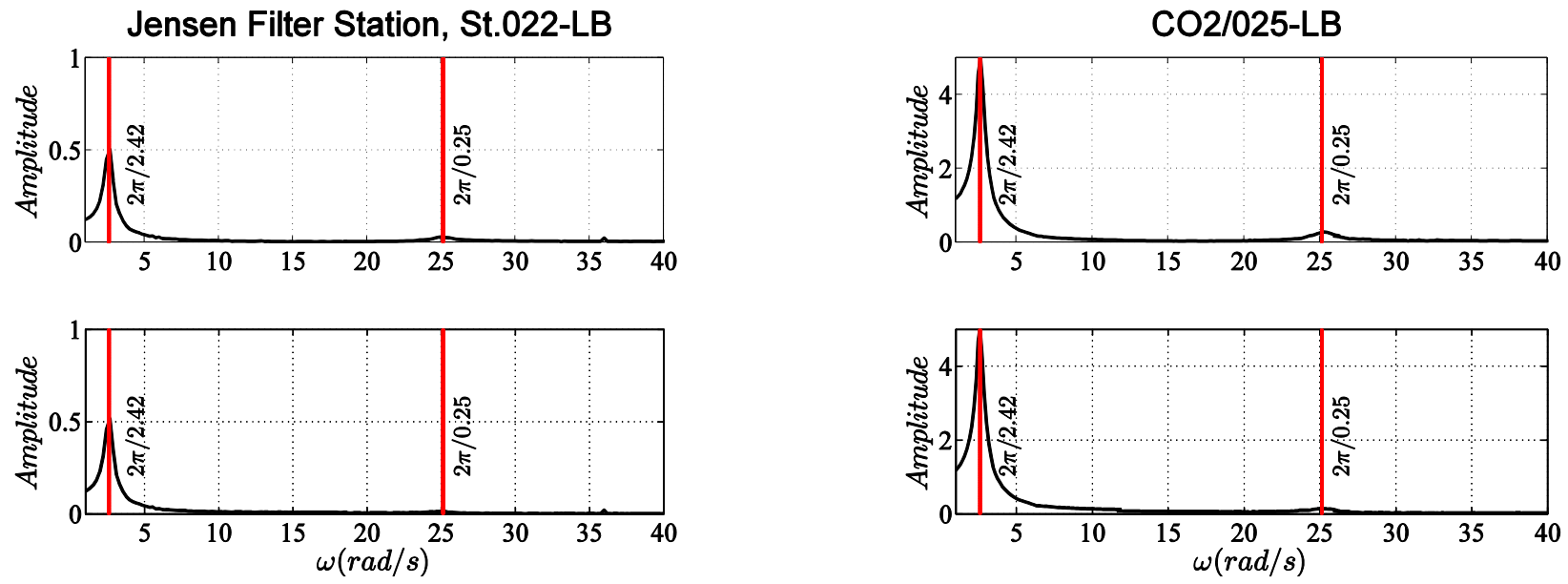

Jensen Filter Station, St.022-LRB
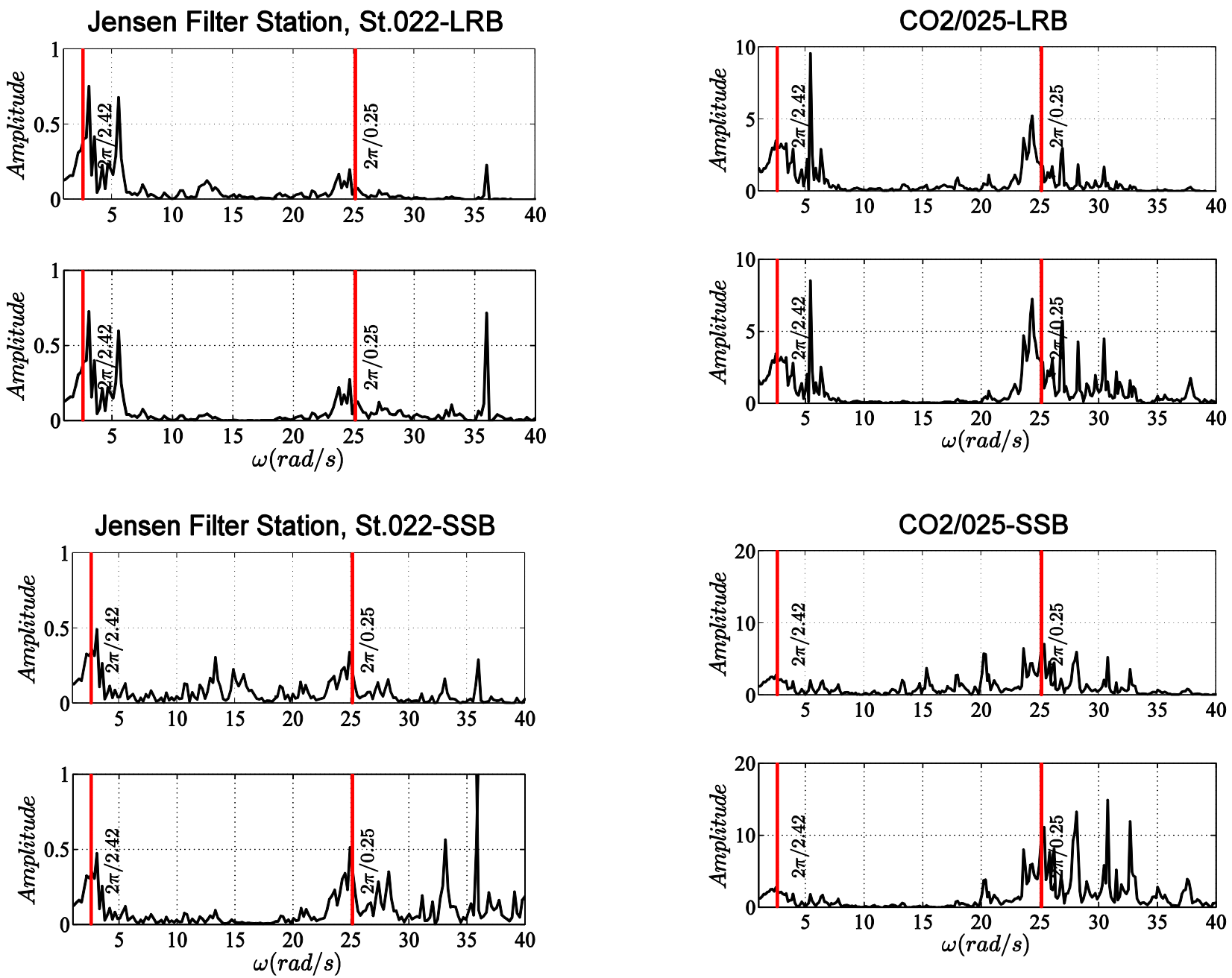

Figure 9. Transfer functions of the 2DOF system isolated by linear (top), lead rubber (middle) and spherical sliding bearings (bottom) when subjected to the Northridge (left) and Parkfield (right) earthquake.

is widely used in wavelet analysis.

In order for a wavelike function to be classified as a wavelet, the wavelike function must have (Addison 2002) : (a) finite energy 


$$
E=\int_{-\infty}^{\infty}|\psi(t)|^{2} d t<\infty
$$

and (b) a zero mean. In this work we are merely interested to achieve the best local matching of any given acceleration record with a wavelet that will offer the best estimates of the period ( $T_{p}=$ time scale $)$ and amplitude $\left(a_{p}\right.$, since $a_{p} T_{p}^{2}=$ length scale) of the prevailing energetic pulse. Accordingly, we perform a series of inner products (convolutions) of the response acceleration signal, $\ddot{u}(t)$, with the wavelet $\psi(t)$ by manipulating the wavelet through a process of translation (i.e. movement along the time axis) and a process of dilation-contraction (i.e. spreading out or squeezing of the wavelet)

$$
C(s, \xi)=w(s) \int_{-\infty}^{\infty} \ddot{u}_{g}(t) \psi\left(\frac{t-\xi}{s}\right) d t
$$

The values of $s=S$ and $\xi=\Xi$, for which the coefficient, $C(s, \xi)=C(S, \Xi)$ becomes maximum offer the scale and location of the wavelet $w(s) \psi\left(\frac{t-\xi}{s}\right)$ that locally best matches the acceleration record, $\ddot{u}(t)$. Equation (24) is the definition of the wavelet transform. The quantity $w(s)$ outside the integral in equation (24) is a weighting function. Typically $w(s)$ is set equal to $1 / \sqrt{s}$ in order to ensure that all wavelets $\psi_{s, \xi}(t)=w(s) \psi\left(\frac{t-\xi}{s}\right)$ at every scale $s$ have the same energy, and according to equation (23)

$$
\int_{-\infty}^{\infty}\left|\psi_{s, \xi}(t)\right|^{2} d t=\int_{-\infty}^{\infty}\left|\frac{1}{\sqrt{s}} \psi\left(\frac{t-\xi}{s}\right)\right|^{2} d t=\left\|\psi_{s, \xi}(t)\right\|_{2}=\text { constant }, \quad \forall s
$$

The same energy requirement among all the daughter wavelets $\psi_{s, \xi}(t)$ is the default setting in the MATLAB wavelet toolbox and has been used by Baker (2007); however, the same energy requirement is, by all means, not a restriction. Clearly there are applications where it is more appropriate that all daughter wavelets $\psi_{s, \xi}(t)$ at every scale $s$ enclose the same area $(w(s)=1 / s)$ or have the same maximum value $(w(s)=1)$. However, in this paper there is no particular need for not using the default same energy requirement for the daughter wavelets.

Figure 10 presents the acceleration response of the 2 dof oscillator when subjected to the 95 Erzincan record of the 1992 Erzincan earthquake. The heavy dashed line presents the wavelet 


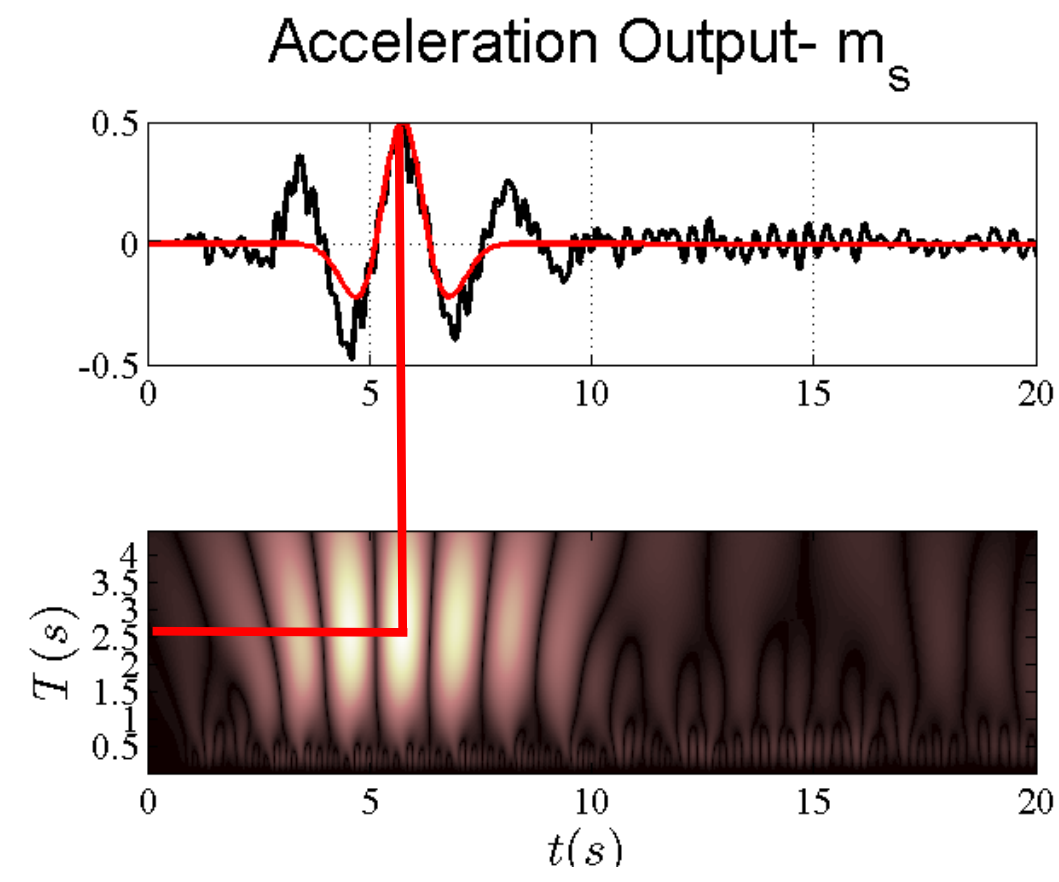

Acceleration Output- $m_{b}$

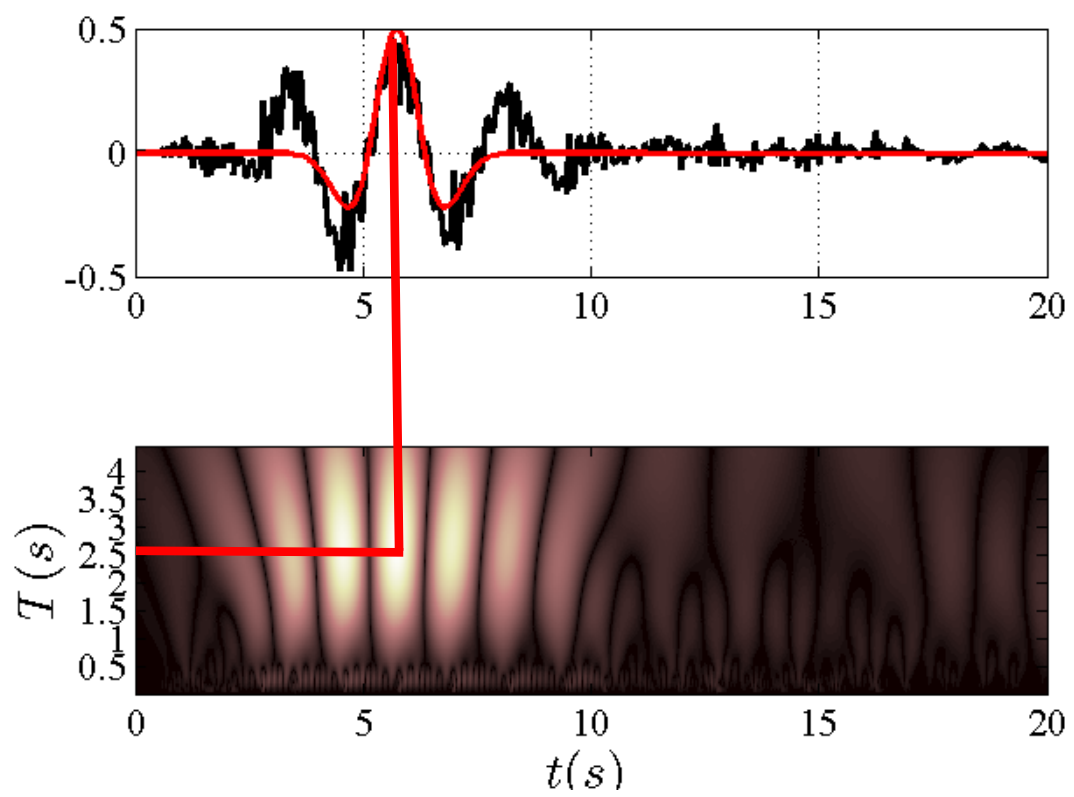

Figure 10. Best fitted wavelets on the acceleration histories above isolators (bottom) and the superstructure (top) when the 2DOF system is subjected to the 1992 Erzincan earthquake together with the associated scalograms.

$\lambda(S, \Xi) w(S) \psi\left(\frac{t-\Xi}{S}\right)$ in which $\Xi$ and $S$ are the values of $\xi$ and $s$ that give the maximum coefficient $C(S, \Xi)$ from equation (24) in which $w(s)=1 / \sqrt{s}$. The multiplication factor

$$
\lambda(S, \Xi)=\frac{C(S, \Xi)}{w^{2}(s) \cdot S \cdot E}
$$


where $E$ is the energy of the mother wavelet, is needed in order for the best matching wavelet, $\psi_{S, \Xi}(t)=w(s) \psi\left(\frac{t-\Xi}{S}\right)$, to assume locally the amplitude of the acceleration record. The wavelets used in this paper are the Ricker wavelet (Ricker 1943, 1944), "Mexican Hat Wavelet", as shown in equation (22) and the cosine wavelet,

$$
\psi(t)=\cos \left(\frac{2 \pi}{T} t\right), \quad 0 \leq t \leq T
$$

However, due to the strict mathematical structure of the wavelets, an extended wavelet transform, advanced by Vassiliou and Makris (2011), had to be applied in order to create a more flexible wavelet transform so it can match better the recorded signals that correspond to non-linear systems such as seismically isolated structures.

In the classical wavelet transform defined with equation (24) the mother wavelet is only subjected to a translation together with a dilation-contraction, $\psi\left(\frac{t-\xi}{s}\right)$. The dilation contraction is controlled with the scale parameter $s$; while, the movement of the wavelet along the time axis is controlled with the translation time, $\xi$. For instance, any daughter wavelet of the symmetric Ricker mother wavelet given by equation (22) assumes the form

$$
\psi\left(\frac{t-\xi}{s}\right)=\left[1-\left(\frac{t-\xi}{s}\right)^{2}\right] e^{-\frac{1}{2}\left(\frac{t-\xi}{s}\right)^{2}}
$$

The need to include four parameters in a mathematical expression of a simple wavelike function has been presented and addressed by Mavroeidis and Papageorgiou (2003). They identified as the most appropriate analytical expression the Gabor (1946) "elementary signal" which they slightly modified to facilitate derivations of closed-form expressions of the spectral characteristics of the signal and response spectra. The Gabor (1946) "elementary signal" is defined as

$$
g(t)=e^{-\left(\frac{2 \pi f_{p}}{\gamma}\right)^{2} t^{2}} \cos \left[2 \pi f_{p} t+\varphi\right]
$$

which is merely the product of a harmonic oscillation with a Gaussian envelop. In equation (29) , $f_{p}$ is the frequency of the harmonic oscillation, $\varphi$ is the phase angle and $\gamma$ is a parameter that controls the oscillations characters of the signal. The Gabor wavelike signal given by 
equation (29) does not have a zero mean; therefore, it cannot be a wavelet within the context of the wavelet transformation.

Nevertheless, the elementary signal proposed by Mavroeidis and Papageorgiou (2003) to approximate velocity pulses is a slight modification of the Gabor signal given by equation (29) where the Gaussian envelope has been replaced by an elevated cosine function.

$$
v(t)=\frac{1}{2}\left(1+\cos \left(\frac{2 \pi f_{p}}{\gamma} t\right)\right) \cos \left(2 \pi f_{p} t+\varphi\right)
$$

Clearly the wavelike signal given by equation (30) does not always have a zero mean; therefore it cannot be a wavelet within the context of wavelet transform. Nevertheless, the time derivative of the elementary velocity signal given by equation (30)

$$
\frac{d v(t)}{d t}=\frac{\pi f_{p}}{\gamma}\left(\sin \left(\frac{2 \pi f_{p}}{\gamma} t\right) \cos \left(2 \pi f_{p} t+\varphi\right)+\gamma \sin \left(2 \pi f_{p} t+\varphi\right)\left(1+\cos \left(\frac{2 \pi f_{p}}{\gamma} t\right)\right)\right)
$$

is by construction a zero-mean signal and is defined in this paper as the Mavroeidis and Papageorgiou (M\&P) wavelet. After replacing the oscillatory frequency, $f_{p}$, with the inverse of the scale parameter the M\&P wavelet is defined as

$$
\psi\left(\frac{t-\xi}{s}, \gamma, \varphi\right)=\left(\sin \left(\frac{2 \pi}{s \gamma}(t-\xi)\right) \cos \left(\frac{2 \pi}{s}(t-\xi)+\varphi\right)+\gamma \sin \left(\frac{2 \pi}{s}(t-\xi)+\varphi\right)\right)\left(1+\cos \left(\frac{2 \pi}{\gamma s}(t-\xi)\right)\right)
$$

The novel attraction in the M\&P wavelet given by equation (32) is that in addition to the dilation-contraction and translation $\left(\frac{t-\xi}{s}\right)$, the wavelet can be further manipulated by modulating the phase, $\varphi$, and the parameter $\gamma$, which controls the oscillatory character (number of half cycles). We can now define the four parameter wavelet transform as

$$
C(s, \xi, \gamma, \varphi)=w(s, \gamma, \varphi) \int_{-\infty}^{\infty} \ddot{u}_{g}(t) \psi\left(\frac{t-\xi}{s}, \gamma, \varphi\right) d t
$$

The inner product given by equation (33) is performed repeatedly by scanning not only all times, $\xi$, and scales, $s$, but also by scanning various phases $\varphi=\{0, \pi / 4, \pi / 2,3 \pi / 4\}$ and various values of the oscillatory nature of the signal $\gamma=\{1,1.5,2,2.5,3\}$. The quantity 
$w(s, \gamma, \varphi)$ outside the integral is a weighting function which is adjusted according to the application.

The values of $s=S, \quad \xi=\Xi, \quad \gamma=\Gamma, \quad \varphi=\Phi$ for which the coefficient $C(s, \xi, \gamma, \varphi)=C(S, \Xi, \Gamma, \Phi)$ becomes maximum offer the scale, location, phase and number of half cycles of the wavelet $\psi\left(\frac{t-\xi}{s}, \gamma, \varphi\right)$ that locally matches best the acceleration record, $\ddot{u}(t)$.

The multiplication coefficient $\lambda(S, \Xi, \Gamma, \Phi)$, which dictates how much the best matching generalized wavelet $w(S, \Gamma, \Phi) \cdot \psi_{S, \Xi, \Gamma, \Phi}(t)$ needs to be amplified to best approximate the energetic acceleration pulse, is obtained with an analysis similar to the one given in Appendix A of Vassiliou and Makris (2011).

$$
\lambda(S, \Xi, \Gamma, \Phi)=\frac{C(S, \Xi, \Gamma, \Phi)}{w^{2}(S, \Gamma, \Phi) \cdot S \cdot E(\Gamma, \Phi)}
$$

\subsection{IDENTIFICATION OF MODAL PROPERTIES OF THE 2DOF SYSTEM WITH WTM}

Figure 11 compares the identified (most energetic) period that prevails in the response of the seismic isolated structure shown in Figure 1 with the theoretical value $\left(T_{1}=2.42 s\right)$.

From Figure 11 it is clear that the WTM identifies satisfactorily the period of isolated structure for all cases both with the Mexican Hat and the M\&P wavelets. The M\&P wavelets give slightly more accurate results due to the mathematical flexibility of the extended transform; a result that may be quite useful in more complex and realistic structures.

\section{Conclusions}

This paper examines the effectiveness of widely used linear identification methods both in time and frequency domain to identify the response of seismically isolated structures supported on bearings with bilinear behavior. A $2 \mathrm{DOF}$ system isolated by linear, lead rubber and spherical sliding bearings has been subjected to four different acceleration time histories listed in Table 2 . 

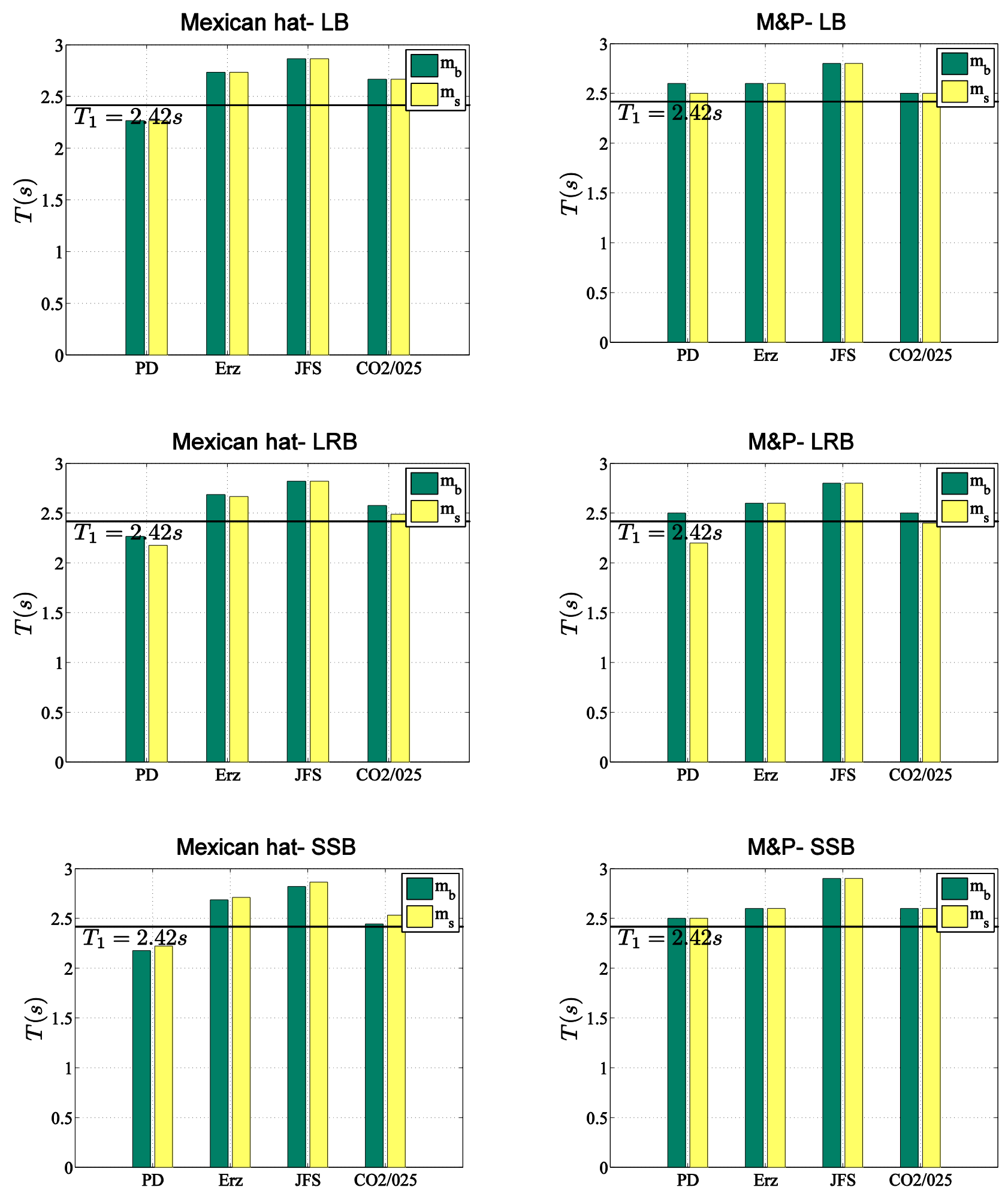

Figure 11. Estimation with wavelet analysis of the period of isolated structure of the 2DOF system when subjected to the four ground motions listed in Table 2.

The Prediction Error Method (PEM) identifies with remarkable accuracy the eigenperiods and modal damping ratios in the case of linear bearings showing the efficiency of the method when 
applied in linear systems. However, in the case where the 2DOF system is isolated by lead rubber and spherical sliding bearings, the PEM is inappropriate to identify the period of isolated structure and the damping ratios of the system.

Moreover, the PPM identifies with accuracy the modal parameters of the linear system as the transfer function exhibit well separated modes. On the other hand, when the 2dof is isolated by lead rubber and spherical sliding bearings the mild nonlinearities of the system are responsible for spurious-noisy spikes in the frequency domain, rendering the PPM impractical.

In an effort to overcome the aforementioned challenges, the paper proceeds with a timefrequency domain analysis using the wavelet transform method (WTM) to back-figure the isolation period of the system.

In conclusion, when the $2 \mathrm{DOF}$ system is isolated by linear isolation bearings the PEM (time domain) and PPM (frequency domain) identify remarkably well the modal parameters of the system. When the 2DOF system is isolated by bearings with bilinear behavior both PEM and PPM show poor performance in back-figuring the period of isolated structure of the system. In contrast, the extended Wavelet transform method (WTM) which employs the M\&P wavelets identifies satisfactorily the period of isolated structure of the structure.

\section{Acknowledgements}

This research has been co-financed by the European Union (European Social Fund - ESF) and Greek national funds through the Operational Program "Education and Lifelong Learning" of the National Strategic Reference Framework (NSRF) - Research Funding Program: Heracleitus II. Investing in knowledge society through the European Social Fund.

\section{References}

1. Addison P. S. (2002), The illustrated wavelet transform handbook. Institute of Physics Handbook.

2. Adeli, H., Jiang, X. (2005), "Dynamic Fuzzy Wavelet Neural Network Model for Structural System Identification", Journal of Structural Engineering.

3. Akira M. (2003), Structural Dynamics for Health Monitoring, SANKEISHA Co., Ltd.

4. Ali, Md, R, and Okabayashi, T, (2011), "System Identification of highway bridges from ambient vibration using subspace stochastic realization theories.", Earthquakes and Structures, 2(2), 189206.

5. Allemang R., J., and Brown D., L., (1998), “A Unified Matrix Polynomial Approach to Modal Identification", Journal of Sound and Vibration, 211(3), 301-322. 
6. “Anti-seismic devices". (2009), European Standard, FprEN 15129, Eurocode.

7. Arici, Y., and Mosalam, K. M. (2005), "Modal Identification of bridge systems using state-space methods", Struct.l Control and Health Monitoring, 12, 381-404.

8. Arici, Y., and Mosalam, K. M. (2006), "Modal Identification and Health Monitoring of Bridges using Seismic Acceleration Records”, EERC 2006-02.

9. Aström, K. J., and Bohlin T. (1965), "Numerical identification of linear dynamic systems from normal operating records", IF AC Symposium on Self-Adaptive Systems, Teddington, England.

10. Baker, W.J., (2007), "Quantitative classification of near fault ground motions using wavelet analysis", Bull. Seism. Soc. Am., 97, 1486-1501.

11. Beck R.T., Beck, J.L. (1985), Comparison between transfer function and modal minimization methods for system identifation, EERC 85-06.

12. Constantinou M.C., Mokha, A.S., Reinhorn, A.M., (1990). "Teflon bearing in base isolation. II: modeling", Journal of Structural Engineering (ASCE), 116:455-474.

13. "Design of lead-rubber bearings".(1983) Civil division publication 818/A, New Zealand Ministry of Works and Development, Wellington, New Zealand.

14. Ewins, D. J. (1984), Modal Testing: Theory and Practice, UK: Research Studies Press.

15. FEMA 310, (1998), Handbook for the Seismic Evaluation of Buildings - A Prestandard, ASCE.

16. Gabor, D. (1946), "Theory of communication. I. The analysis of information”, IEEE 93, 429441.

17. Guide specifications for seismic isolation design, (1991). American Association of State Highway and Transportation officials, Washington, D.C.

18. Hearn, G., Testa, R., B. (1991), "Modal analysis for damage detection in structures", Journal of Structural Engineering; 117(10), 3042-3063.

19. Juang J.N. (1994), Applied system identification. Prentice Hall, PTR: New Jersey.

20. Kelly, J., M. (1997), Earthquake-Resistant Design with Rubber. Springer: London, U.K.

21. Kim, H., Melhem, H. (2003), "Damage detection of structures by wavelet analysis", Engineering Structures, 26(3),347-362.

22. Lembregts, F., Leuridan, J., Van Brussel, H. (1990), "Frequency Domain Direct Parameter Identification for Modal Analysis: State Space Formulation", Mechanical Systems and Signal Processing, 4: 65-75.

23. Ljung L. (1987), System Identification-Theory for the User, Prentice-Hall, New Jersey.

24. Ljung L. (1994), "State of the Art in Linear System Identification: Time and Frequency Domain Methods", Proceedings of '04 American Control Conference, 1, 650-660.

25. Ljung L. (2002), "Prediction Error Estimation Methods", Circuits Systems Signal Processing, 21: 1121.

26. Maia, N. M. M., and Silva, J. M. M. (2001) "Modal analysis identification techniques", Phil.Trans. R. Soc. Lond., 359, 29-40.

27. Maia, N. M. M. (2001), "Parameter extraction methods", Modal Analysis, Experimental, 820-824 (doi:10.1006/rwvb.2001.0028).

28. Makris, N., and Black, C. J. (2004a), "Dimensional analysis of rigid-plastic and elastoplastic structures under pulse-type excitations", J.Eng. Mech., 130, 1006-1018.

29. Makris, N., and Black, C. J. (2004b), "Dimensional analysis of bilinear oscillators under pulsetype excitations", J. Eng. Mech., 130, 1019-1031.

30. Makris N., and C. Black (2004c), "Evaluation of peak ground velocity as a "good" intensity measure for near-source ground motions", J. Eng. Mech., ASCE 130, 1032-1044.

31. Makris N, Chang S. (2000) "Effect of viscous, viscoplastic and friction damping on the response of seismic isolated structures". Journal of Earthquake Engineering and Structural Dynamics, 29(1): 85-107.

32. Makris N., and Vassiliou M. F., (2011) 'The existence of 'complete. similarities' in the response of seismic isolated structures subjected to pulse-like ground motions and their implications in analysis", Journal of Earthquake Engineering and Structural Dynamics, 40(10),1103-1121.

33. Mallat S. G. (1999). A wavelet tour of signal processing. Academic Press.

34. MATLAB. High-performance Language Software for Technical Computation. The MathWorks, Inc: Natick, MA, 2002. 
35. Mavroeidis, G. P., A. S. Papageorgiou (2003), "A mathematical representation of near-fault ground motions", Bull. Seism. Soc. Am., 93, 1099-1131.

36. Mavroeidis GP, G. Dong, AS. Papageorgiou (2004), "Near-fault ground motions, and the response of elastic and inelastic single-degree-of-freedom (SDOF) systems", Earthquake Engineering and Structural Dynamics, 33, 1023-1049.

37. Mokha, A.S., Constantinou, M.C., Reinhorn, A.M., (1990), "Teflon bearing in base isolation. I: testing", Journal of Structural Engineering (ASCE), 116: 438-454.

38. Olmos,B.A., and Roesset, J.M., (2010), "Effects of the nonlinear behavior of lead-rubber bearings on the seismic response of bridges", Earthquakes and Structures, 1(2),215-230.

39. Peeters, B., Ventura, C.E. (2003), "Comparative study of Modal Analysis techniques for bridge dynamic characteristics", Mechanical Systems and Signal Processing, 17, 965-988.

40. Ricker, N. (1943), "Further developments in the wavelet theory of seismogram structure", Bull. Seism. Soc. Am., 33, 197-228.

41. Ricker, N. (1944), "Wavelet functions and their polynomials", Geophysics 9, 314-323.

42. Salawu, O., S. (1997), "Detection of structural damage through changes in frequency: a review", Engineering Structures, 19, 718-723.

43. Shrikhande, M., (2011), "Reconstruction of missing response data for identification of higher modes", Earthquakes and Structures, 2(4), 323-336.

44. Vassiliou, M., F., and Makris, N., (2011), "Estimating Time Scales and Length Scales in Pulselike Earthquake Acceleration Records with Wavelet Analysis", BSSA, 101(2), 596618.

45. Wang, Z., Lin, R., M., Lim, M., K. (1997), "Structural damage detection using measured FRF data", Comput. Methods Appl. Mech. Engrg., 147, 187-197.

\section{List of captions}

Figure 1. A one bay frame supported on a seismic isolated base.

Figure 2. Schematic of a force-displacement loop with bilinear behavior.

Figure 3. Ground, above isolators and superstructure acceleration histories together with the corresponding force-displacement loops of the 2DOF system shown in Figure 1 supported on lead rubber bearings when subjected to the four recorded motions listed in Table 2.

Figure 4. Ground, above isolators and superstructure acceleration histories together with the corresponding force-displacement loops of the 2DOF system shown in Figure 1 supported on spherical sliding bearings when subjected to the four recorded motions listed in Table 2.

Figure 5. Eigenperiods and damping ratios of the 2DOF system shown in Figure 1 when isolated by linear viscoelastic bearings identified with the Prediction Error Method (PEM).

Figure 6. Eigenperiods and damping ratios of the 2DOF system shown in Figure 1 when isolated by lead rubber bearings identified with the Prediction Error Method (PEM).

Figure 7. Eigenperiods and damping ratios of the 2DOF system shown in Figure 1 when isolated by spherical sliding bearings identified with the Prediction Error Method (PEM). 
Figure 8. Transfer functions of the $2 \mathrm{DOF}$ system isolated by linear (top), lead rubber (middle) and spherical sliding bearings (bottom) when subjected to the San Fernando (left) and Erzincan (right) earthquake.

Figure 9. Transfer functions of the $2 \mathrm{DOF}$ system isolated by linear (top), lead rubber (middle) and spherical sliding bearings (bottom) when subjected to the Northridge (left) and Parkfield (right) earthquake.

Figure 10. Best fitted wavelets on the acceleration histories above isolators (bottom) and the superstructure (top) when the 2DOF system is subjected to the 1992 Erzincan earthquake together with the associated scalograms.

Figure 11. Estimation with wavelet analysis of the period of isolated structure of the 2DOF system when subjected to the four ground motions listed in Table 2. 